\title{
Matchings in Random Biregular Bipartite Graphs
}

\author{
Guillem Perarnau* \\ Departament de Matemàtica Aplicada $I V$ \\ Universitat Politècnica de Catalunya, BarcelonaTech \\ Barcelona, Spain \\ guillem.perarnau@ma4.upc.edu
}

\author{
Giorgis Petridis \\ Department of Mathematics \\ University of Rochester \\ Rochester, NY 14627, USA. \\ giorgis@cantab.net
}

Submitted: Aug 24, 2012; Accepted: Mar 10, 2013; Published: Mar 24, 2013

Mathematics Subject Classifications: 05C80, 05D40, 11B13

\begin{abstract}
We study the existence of perfect matchings in suitably chosen induced subgraphs of random biregular bipartite graphs. We prove a result similar to a classical theorem of Erdős and Rényi about perfect matchings in random bipartite graphs. We also present an application to commutative graphs, a class of graphs that are featured in additive number theory.
\end{abstract}

Keywords: Random biregular bipartite graphs, Perfect matchings, Commutative graphs.

\section{Introduction}

Let us begin by defining the terms that appear in the title. Recall that, given two sets $A$ and $B$ of equal size and a bipartite directed graph on vertex set $(A, B)$, a perfect matching (also known as a 1 -factor) from $A$ to $B$ is a collection of $|A|$ vertex disjoint edges from $A$ to $B$.

Definition 1. Let $k \in \mathbb{Q}^{+}$be a positive rational number, $n \in \mathbb{Z}^{+}$a positive integer that satisfies $k n \in \mathbb{Z}^{+}$and $d \in \mathbb{Z}^{+}$a positive integer that satisfies $1 \leqslant d \leqslant n$ and $k d \in \mathbb{Z}^{+}$. Let $Y$ be a set of size $n$ and $Z$ be a set of size $k n$. Define $\mathcal{G}(k, n, d)$ to be the family of biregular bipartite directed labelled graphs on the vertex set $(Y, Z)$ (with edges directed from $Y$ to $Z$ ) where $d^{+}(y)=k d$ for all $y \in Y$ and $d^{-}(z)=d$ for all $z \in Z$. A random biregular bipartite directed graph (with parameters $k, n, d$ ) is a graph chosen from $\mathcal{G}(k, n, d)$ uniformly at random. This probability space of random graphs is denoted by $G(k, n, d)$.

*This work has been partially supported by the Catalan Research Council under grant 2009SGR01387 and the Spanish Council under project MTM2011-28800-C02-01. Eventually, the author wants to thank the FPU grant from the Ministerio de Educación de España. 
The family $\mathcal{G}(k, n, d)$ is non-empty. We illustrate this by giving an example for integer $k$, which is indicative of how biregular bipartite graphs are featured in additive number theory. We identify $Z$ with $\mathbb{Z}_{k n}$ and $Y$ with the subgroup $\{0, k, 2 k, \ldots,(n-1) k\}$. For $y \in Y$ and $z \in Z$ we place an edge $y z \in E(G)$ if $z-y \in\{0,1, \ldots k d-1\} \bmod (k n)$. The resulting graph is a member of $\mathcal{G}(k, n, d)$.

The case where $k=1$ has a special relevance since $\mathcal{G}(1, n, d)$ is the family of regular bipartite graphs of size $n$ and degree $d$ where the edges are canonically oriented from one stable set to the other. Estimating the size of $\mathcal{G}(1, n, d)$ as a function of $d$ and $n$ is a question that has been studied extensively [8, 21]. Generalizations of this problem to biregular bipartite graphs [19,3] as well as to graphs with a prescribed sequence of degrees in each of the stables have also been studied [17, 18].

Using Hall's theorem it is straightforward to check that every member of $\mathcal{G}(1, n, d)$ has a perfect matching (see e.g. [6, Corollary 2.1.3]). For members of $\mathcal{G}(k, n, d)$ with $k \neq 1$ there can be no perfect matching as the size of the two stable sets is not equal. The distribution of the number of perfect matchings in random regular bipartite graphs was studied by Bollobás and McKay in [2], where its expected value and variance are determined.

We tackle a different kind of question by studying the existence of a perfect matching in induced subgraphs $H$ of members of $\mathcal{G}(k, n, d)$, whose stable sets have equal size. In particular we determine how the probability of having such a perfect matching changes with $d$. Our result is analogous to a classical result of Erdős and Rényi.

Before stating the main result of the paper we recall that in any model of random graphs a property holds with high probability if the probability that a random graph in the model satisfies this property tends to 1 as $n$ tends to infinity. From now on the phrase will be abbreviated to whp, as it is common in the literature.

Theorem 2. Let $k \in \mathbb{Q}^{+}, n \in \mathbb{Z}^{+}$be arbitrarily large and $d \in\{1, \ldots, n\}$ and suppose that $k n, k d \in \mathbb{Z}^{+}$with $k d \leqslant n$.

Furthermore let $Y$ and $Z$ be sets of size respectively $n$ and $k n$, and take subsets $A \subseteq Y$ and $B \subseteq Z$ of size $k d$. Let $G \sim G(k, n, d)$ and define $H:=G[A, B]$ to be the subgraph induced by $G$ on vertex set $(A, B)$. Then

(i) No perfect matching exists in $H$ whp when $\frac{k d^{2}}{n}-\log (k d) \rightarrow-\infty$ or when $d$ is a constant.

(ii) A perfect matching exists in $H$ whp when $\frac{k d^{2}}{n}-\log (k d) \rightarrow+\infty$.

Remark 3. The second condition in conclusion $(i)$ has to be included because when $d$ is constant the quantity $\frac{k d^{2}}{n}-\log (k d)$ does not tend to $-\infty$.

Here and elsewhere, for any $y \in Y$ we define $\Gamma(y)=\{z \in Z: y z \in E(G)\}$ and for any $S \subseteq Y, \Gamma(S)=\cup_{y \in S} \Gamma(y)$. Similarly we define the inverse neighbourhood of $z \in Z$ by $\Gamma^{-1}(z)$ and the inverse neighbouhood of $T \subseteq Z$ by $\Gamma^{-1}(T)$.

The next result is a variation of Theorem 2 when $B=\Gamma(y)$ for some $y \in A$. 
Theorem 4. Let $k \in \mathbb{Q}^{+}, n \in \mathbb{Z}^{+}$be arbitrarily large and $d \in\{2, \ldots, n\}$ and suppose that $k n, k d \in \mathbb{Z}^{+}$with $k d \leqslant n$.

Furthermore let $Y$ and $Z$ be sets of size respectively $n$ and $k n$ and $G \sim G(k, n, d)$. Take a subset $A \subseteq Y$ of size $k d$ and $y \in A$. Define $H:=G[A, \Gamma(y)]$ to be the subgraph induced by $G$ on vertex set $(A, \Gamma(y))$. Then

(i) No perfect matching exists in $H$ whp when $\frac{k d^{2}}{n}-\log (k d) \rightarrow-\infty$ or when $d$ is a constant.

(ii) A perfect matching exists in $H$ whp when $\frac{k d^{2}}{n}-\log (k d) \rightarrow+\infty$.

The case $d=1$ is not covered by Theorem 4 . It is nonetheless easy to check that for $d=1$ a perfect matching exists if and only if $k=1$.

To put our results in context we briefly describe what holds in the most standard model of random directed bipartite graphs.

Definition 5. Let $A$ and $B$ be two sets of size $n$. A random bipartite graph with parameters $n, p$ is a bipartite graph on the vertex set $(A, B)$ where edges are chosen independently of each other with probability $p$. The model of random bipartite graphs is denoted by $B(n, p)$.

The existence of perfect matchings in random bipartite graphs was investigated by Erdős and Rényi about fifty years ago. They established the following in [7].

Theorem 6 (Erdős-Rényi). Let c be a constant and $n$ an arbitrarily large positive integer. Furthermore let

$$
p=\frac{\log n+c}{n}
$$

and consider a random bipartite graph $G^{\prime} \sim B(n, p)$.

Then the probability that $G^{\prime}$ contains a perfect matching is asymptotically equal to $\exp \left(-2 e^{-c}\right)$.

In particular if $n p-\log n \rightarrow+\infty$ when $n \rightarrow+\infty$, then there exists a matching in $G^{\prime}$ whp; and if $n p-\log n \rightarrow-\infty$ when $n \rightarrow+\infty$, then no perfect matching exists in $G^{\prime}$ whp.

Theorem 2 is an Erdős-Rényi type result for the induced subgraph $H$. To make the similarity between Theorem 2 and Theorem 6 as clear as possible we set $k=1$ in the former. The induced subgraph $H$ is somewhat similar to a random bipartite graph as it has similar properties to $G^{\prime} \sim B(d, d / n)$ : The size of the stables of $H$ is $d$ and edges appear in ( $G$ and hence also in) $H$ with uniform probability $d / n$. The main difference is that edges do not appear independently in $H$, yet the dependence is generally speaking small. The similarity between $H$ and $G^{\prime}$ is reflected by the fact that a perfect matching exists in both graphs whp when $d^{2} / n-\log d \rightarrow+\infty$.

A related question that has been studied more extensively concerns not necessarily bipartite graphs. The models under consideration are $G_{d}(n)$ (a graph chosen uniformly at random from all $d$-regular graphs on $n$ vertices) and $G(n, p)$ (a graph on $n$ vertices where edges are chosen independently with probability $p$ ) where $p=d / n$. Two kinds or 
results have been obtained. On one hand, properties of graphs that hold whp in $G(n, p)$ have been shown to also hold $w h p$ in $G_{d}(n)[13,11,12]$. On the other hand, Kim and Vu have studied the contiguity of both models in [10]. They conjectured that the $G_{d}(n)$ is contiguous to the $G(n, p)$ when $d \gg \log n$ (Sandwich conjecture), but were only able to show a slightly weaker relation between the models when $d \ll n^{1 / 3} / \log ^{2} n$ (their method can be applied in some cases to larger values of $d$ up to $d=o(\sqrt{n})$ ). If their result could be extended to $d$ in the $\sqrt{n \log n}$ range (and also to bipartite graphs) it would imply that the induced subgraph $H$ and $G^{\prime} \sim B(d, d / n)$ are also contiguous, giving a straightforward proof of Theorem 2 as a corollary of Theorem 6 .

The main motivation to study the existence of perfect matchings in induced subgraphs of random biregular bipartite graphs has to do with commutative graphs and Plünnecke's inequality. A comprehensive study of the applications of commutative graphs and Plünnecke's inequality can be found in [26]. Here we only present the necessary facts that relate commutative graphs with Theorem 4 . We begin with two definitions definition.

Definition 7. A directed graph $G$ with vertex set $V(G)=X_{0} \cup X_{1} \cup \cdots \cup X_{h}$ is called layered (where the $X_{i}$ are the layers) if there are edges only between consecutive layers, so that $E\left(X_{i}, X_{j}\right)=\emptyset$ unless $j=i+1$ for all $0 \leqslant i, j \leqslant h$.

Definition 8. A directed layered graph $G$ with layers $X_{0}, X_{1}, \ldots, X_{h}$ is called commutative if

1. For all $1 \leqslant i \leqslant h-1$ and $u v \in E\left(X_{i-1}, X_{i}\right)$ there exists a perfect matching from a subset of $\Gamma(u)$ to $\Gamma(v)$. This condition is called Plünnecke's upward condition (PU).

2. For all $1 \leqslant i \leqslant h-1$ and $u v \in E\left(X_{i}, X_{i+1}\right)$ there exists a perfect matching from a subset of $\Gamma^{-1}(v)$ to $\Gamma^{-1}(u)$. This condition is called Plünnecke's downward condition $(P D)$.

Observe that when $G$ is biregular the perfect matching in Plünnecke's upward (downward) condition is from the whole $\Gamma(u)$ to $\Gamma(v)\left(\Gamma^{-1}(v)\right.$ to $\left.\Gamma^{-1}(u)\right)$.

Plünnecke introduced commutative graphs to study the growth of sumsets $[24,25,22$, 27]. He was interested in the magnification ratios of graphs.

$$
D_{i}(G)=\min _{\emptyset \neq Z \subseteq X_{0}} \frac{\left|\Gamma^{(i)}(Z)\right|}{|Z|},
$$

where $1 \leqslant i \leqslant h$ and $\Gamma^{(i)}(Z)$ is defined iteratively by $\Gamma^{(i)}(Z)=\Gamma\left(\Gamma^{(i-1)}(Z)\right)$. Plünnecke proved a powerful inequality that limits the growth of magnification ratios of commutative graphs.

Theorem 9 (Plünnecke). Let $G$ be a commutative graph. Then the sequence $D_{i}(G)^{1 / i}$ is decreasing.

In [23] it was shown that the upper bound for $D_{i}(G) \leqslant D_{1}(G)^{i}$ given by Theorem 9 is sharp. In particular a commutative graph $G$ that satisfies $D_{i}(G)=k^{i}$ for all $1 \leqslant i \leqslant h$ 
was constructed for all $k \in \mathbb{Q}^{+}$and $h \in \mathbb{Z}^{+}$. The extremal examples were biregular commutative graphs whose in and out degrees satisfied $d^{+} / d^{-}=k$. In fact it is easy to check that, in any commutative graph whose degrees satisfy $d^{+} / d^{-}=k$, the sequence $D_{i}(G)^{1 / i}$ is constant and equal to $k$.

We apply Theorem 4 to give a non-constructive, and probabilistic in nature, proof of the existence of graphs that are extremal for Plünnecke's inequality, answering a question of Gowers.

We form a layered directed biregular graph by "placing random biregular bipartite directed graphs on top of each other." If in each biregular bipartite graph that is induced by two consecutive layers, the out-degree of the bottom layer is large enough compared to its size, then the resulting graph is whp commutative.

Theorem 10. Let $1 \leqslant k \in \mathbb{Q}^{+}, m \in \mathbb{Z}^{+}$be arbitrarily large, $d \in\{2, \ldots, m\}$ and $h \in \mathbb{Z}^{+}$. Suppose that $k m, k d \in \mathbb{Z}^{+}$.

Furthermore let $X_{0}, X_{1}, \ldots, X_{h}$ be sets with $\left|X_{i}\right|=k^{i} m$. For $1 \leqslant i \leqslant h$ let $G_{i}:=$ $G_{i}\left[X_{i-1}, X_{i}\right] \sim G\left(k, k^{i-1} m, d\right)$.

Let $G$ be a graph with vertex set $V(G)=X_{0} \cup \cdots \cup X_{h}$ and edge set $E(G)=\cup_{i=1}^{h} E\left(G_{i}\right)$. Then

(i) The graph $G$ is not commutative whp when $d \leqslant \sqrt{\frac{1}{3} k^{h-2} m \log (k m)}$.

(ii) The graph $G$ is commutative whp when $d \geqslant 3 \sqrt{k^{h-2} m \log \left(h k^{h+1} m\right)}$.

Observe that the upper bound and the lower bound in Theorem 10 have the same asymptotic order. We make no effort to optimize the constants as our method does not lead to matching lower and upper bounds.

Results on random regular graphs are usually derived using the so-called configuration (or pairing) model due to Bollobás [1] (for a detailed presentation see [28]). However, this model does not give meaningful results when the degree is large. McKay introduced in [15] a new way to approach problems in random regular graphs when the degree is large, based on switching the edges of the graph. This method has been successfully applied to extend a lot of results for random regular graphs with large degree $[20,13,5,4,11,12]$.

Our strategy is to mirror the proof of Theorem 6 of Erdös and Rényi. The biggest obstacle is dealing with dependencies among the edges. We do this by repeatedly using three ingredients: the regularity of the degrees, the symmetry of $\mathcal{G}(k, n, d)$ and the idea of edge switchings.

The existing estimates on the number of biregular bipartite graphs contain error terms, which are negligible when $d$ is small compared to $n$, but become significant for larger $d$. We will not need to estimate $|\mathcal{G}(k, n, d)|$ and so we will not be affected by this.

The paper is organised as follows. In the next section we introduce the methods we will use repeatedly throughout the paper. In Section 3 we prove a useful result, whose proof demonstrates how the lack of independence in choosing the edges can be overcome. In Section 4 we prove Theorem 4 and in Section 5 we present the backbone of the proof of Theorem 2. Finally in Section 6 we prove Theorem 10. 
Notation. We conclude the introduction with a quick recap of standard notation we will use throughout the paper. The out-degree of a vertex $v$ is $d^{+}(v)=|\Gamma(v)|$ and the minimum out-degree of a directed graph $G$ is $\delta^{+}(G)=\min \left\{d^{+}(v): v \in V(G)\right\}$. The in-degree of a vertex $v$ is similarly defined by $d^{-}(v)=\left|\Gamma^{-1}(v)\right|$ and so is the minimum in-degree $\delta^{-}(G)$ of a directed graph $G$. The minimum degree of a directed graph $G$ is $\delta(G)=\min \left\{\delta^{-}(G), \delta^{+}(G)\right\}$.

For any two functions $f, g$ we write $f(n)=O(g(n))$ if $|f(n)| \leqslant C|g(n)|$ for some absolute constant $C$ and $f(n)=\Theta(g(n))$ if $f(n)=O(g(n))$ and $g(n)=O(f(n))$. We also write $f(n)=o(g(n))$ to mean that $\lim _{n \rightarrow+\infty} f(n) / g(n)=0$. In particular we use $f(n)=o(1)$ if $\lim _{n \rightarrow+\infty} f(n)=0$.

\section{Switching in biregular bipartite graphs}

The first result we prove illustrates how the regularity of the degrees and the symmetry of biregular bipartite graphs will be used in the paper.

Lemma 11. Let $k, n, d$ be like in the statement of Theorem 2. Suppose that $G \sim$ $G(k, n, d)$.

(i) Let $y, y^{\prime} \in Y$. Then

$$
\mathbb{E}\left(\left|\Gamma(y) \cap \Gamma\left(y^{\prime}\right)\right|\right)=\frac{k d(d-1)}{n-1} .
$$

(ii) Let $y \in Y$ and $B \subseteq Z$. Then

$$
\mathbb{E}(|\Gamma(y) \cap B|)=\frac{d|B|}{n} .
$$

Proof. First note that $\Gamma(y)$ is chosen uniformly at random from all $(k d)$-element subsets of $Z$. Without loss of generality we can therefore assume that it is fixed and equal to a set $S$. Next we observe that

$$
\mathbb{E}\left(\left|\Gamma(y) \cap \Gamma\left(y^{\prime}\right)\right|\right)=\sum_{z \in S} \operatorname{Pr}\left(z \in \Gamma\left(y^{\prime}\right)\right) .
$$

The probability $\operatorname{Pr}\left(z \in \Gamma\left(y^{\prime}\right)\right)$ is equal for all $z \in S$. To see why take $z_{0}, z_{1} \in S$ and observe that there exists a bijection $\theta$ from

$$
\mathcal{G}_{z_{0}}=\left\{G \in \mathcal{G}(k, n, d): \Gamma(y)=S \wedge y^{\prime} z_{0} \in E(G)\right\}
$$

to

$$
\mathcal{G}_{z_{1}}=\left\{G \in \mathcal{G}(k, n, d): \Gamma(y)=S \wedge y^{\prime} z_{1} \in E(G)\right\} .
$$

The bijection $\theta$ maps $z_{0}$ to $z_{1}$ and vice versa and restricts to the identity on $V(G) \backslash\left\{z_{0}, z_{1}\right\}$. So

$$
\mathbb{E}\left(\left|\Gamma(y) \cap \Gamma\left(y^{\prime}\right)\right|\right)=k d \operatorname{Pr}\left(z_{0} \in \Gamma\left(y^{\prime}\right)\right) .
$$


On the other hand

$$
\begin{aligned}
d-1 & =\mathbb{E}\left(\left|\Gamma^{-1}\left(z_{0}\right) \backslash\{y\}\right|\right) \\
& =\sum_{v \in Y \backslash\{y\}} \operatorname{Pr}\left(z_{0} \in \Gamma(v)\right) \\
& =(n-1) \operatorname{Pr}\left(z_{0} \in \Gamma\left(y^{\prime}\right)\right) .
\end{aligned}
$$

The third identity following from the symmetry of random biregular bipartite graphs. The first conclusion follows. The second can be proved similarly.

The arguments in the above proof are not sufficient when dealing with more complicated events. To deal with such events we will employ elementary counting arguments that involve switchings.

Definition 12. Let $a, b \in Y$ and $c, d \in Z$ such that $a c, b d \in E(G)$ and $a d, b c \notin E(G)$. The $\{a c, b d\}$-switching of $G$ is the graph $H$ with the same set of vertices as $G$ and $E(H)=$ $E(G) \cup\{a d, b c\} \backslash\{a c, b d\}$.

Figure 1 offers an illustration of this natural operation. Observe that if $G$ is biregular bipartite, then so is $H$; and that if $H$ is the $\{a c, b d\}$-switching of $G$, then $G$ is the $\{a d, b c\}$ switching of $H$. Switchings between graphs were first used by McKay in [15] to obtain
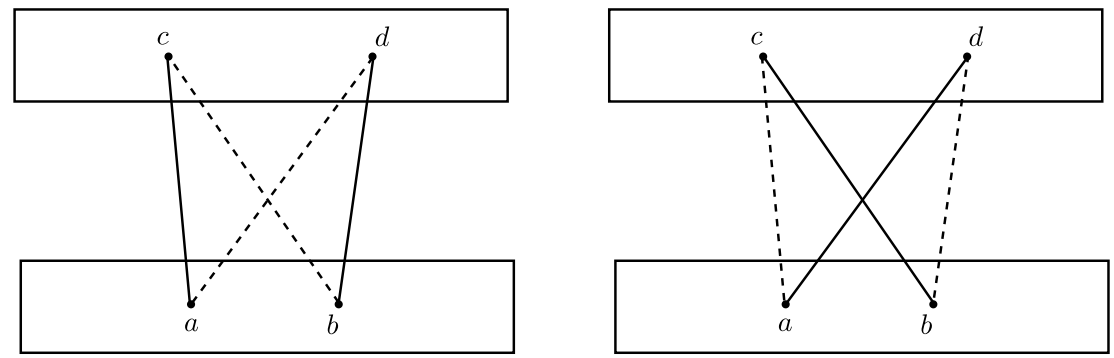

Figure 1: A graph $G$ and its $\{a c, b d\}$-switching $H$. Solid lines represent edges and dashed lines missing edges.

bounds on the probability that a fixed graph appears as a subgraph of a random regular graph. McKay [18] used the same technique to extend the range of $d$ in the enumeration of regular graphs to $d=o\left(n^{1 / 3}\right)$. McKay and Wormald in [20] improved that range to $d=o(\sqrt{n})$ by introducing a new type of switching. Switching is moreover useful in proving that whp regular graphs are expanders [9] or in counting the number of spanning trees subject to an asymptotic condition on the number of cycles [16].

As mentioned in the introduction, switching has more recently been used to study various properties of random regular graphs $[13,5,4,11,12]$. We will use it in a similar fashion to compare the sizes of two families of biregular bipartite graphs, say $\mathcal{G}_{1}$ and $\mathcal{G}_{2}$. We will count in two ways the number of switchings between the two families. In other words we will double count the number of ordered pairs $\left(G_{1}, G_{2}\right) \in \mathcal{G}_{1} \times \mathcal{G}_{2}$ where $G_{1}$ is a switching of $G_{2}$, which is equivalent to $G_{2}$ being a switching of $G_{1}$. 


\section{Preliminary results}

Let $Y, Z$ and $A$ be defined as in Theorem 4. The key to most of the calculations leading to the proof of Theorem 4 is having a good upper bound on the probability that there are no edges between two sets $S \subseteq A$ and $T \subseteq \Gamma(y)$, for some $y \in A$. As $y$ is joined to all vertices in $\Gamma(y)$ we assume that $S \subseteq A \backslash\{y\}$. The main result of this section is the following.

Proposition 13. Let $Y, Z, G$ and $y$ be like in the statement of Theorem 4. Suppose that $T \subseteq \Gamma(y), z_{1} \in T$ and $S \subseteq Y \backslash\{y\}$, where $|S|+d \leqslant n$. Then the probability that there are no edges from $S$ to $T$ is bounded above by:

$$
\begin{aligned}
\operatorname{Pr}(\Gamma(S) \cap T=\emptyset) & \leqslant \operatorname{Pr}\left(\Gamma^{-1}\left(z_{1}\right) \cap S=\emptyset\right)^{|T|} \\
& =\left(1-\frac{|S|}{n-1}\right)^{|T|}\left(1-\frac{|S|}{n-2}\right)^{|T|} \ldots\left(1-\frac{|S|}{n-d+1}\right)^{|T|} \\
& \leqslant \exp \left(-\frac{d|S||T|}{n}\right) .
\end{aligned}
$$

Before giving the proof we quickly present a heuristic explanation for the crucial first inequality. For simplicity we take $T=\left\{z_{1}, z_{2}\right\}$. Suppose for a moment that the neighbourhoods of vertices in $S$ were chosen independently. Then we would have

$$
\operatorname{Pr}(\Gamma(S) \cap T=\emptyset)=\operatorname{Pr}\left(\Gamma^{-1}\left(z_{1}\right) \cap S=\emptyset\right) \operatorname{Pr}\left(\Gamma^{-1}\left(z_{2}\right) \cap S=\emptyset\right)=\operatorname{Pr}\left(\Gamma^{-1}\left(z_{1}\right) \cap S=\emptyset\right)^{2} .
$$

As we do not have independence we have to instead use conditional probabilities:

$$
\operatorname{Pr}(\Gamma(S) \cap T=\emptyset)=\operatorname{Pr}\left(\Gamma^{-1}\left(z_{1}\right) \cap S=\emptyset\right) \operatorname{Pr}\left(\Gamma^{-1}\left(z_{2}\right) \cap S=\emptyset \mid \Gamma^{-1}\left(z_{1}\right) \cap S=\emptyset\right) .
$$

Conditioning on the event $\Gamma^{-1}\left(z_{1}\right) \subseteq Y \backslash S$ has an effect on $Y \backslash S$ : the vertices in $\Gamma^{-1}\left(z_{1}\right)$ have one of the $k d$ edges coming out of them "taken up" by $z_{1}$. One expects that this makes $\Gamma^{-1}\left(z_{2}\right)$ less likely to include them and consequently that

$$
\operatorname{Pr}\left(\Gamma^{-1}\left(z_{2}\right) \cap S=\emptyset \mid \Gamma^{-1}\left(z_{1}\right) \cap S=\emptyset\right) \leqslant \operatorname{Pr}\left(\Gamma^{-1}\left(z_{1}\right) \cap S=\emptyset\right) .
$$

Proving this type of upper bound for conditional probabilities is the main task lying ahead.

Proof of Proposition 13. The second inequality can be proved by induction on $d$ and the third is standard, so we only prove the first inequality and the expression for $\operatorname{Pr}\left(\Gamma^{-1}\left(z_{1}\right) \cap\right.$ $S=\emptyset)$. We let $s=|S|, T=\left\{z_{1}, \ldots, z_{t}\right\}$ and proceed by induction on $t$.

When $t=1$ we have

$$
\operatorname{Pr}\left(\Gamma^{-1}\left(z_{1}\right) \cap S=\emptyset\right)=\frac{\left(\begin{array}{c}
n-1-s \\
d-1
\end{array}\right)}{\left(\begin{array}{l}
n-1 \\
d-1
\end{array}\right)}=\left(1-\frac{s}{n-1}\right)\left(1-\frac{s}{n-2}\right) \ldots\left(1-\frac{s}{n-d+1}\right)
$$


as $\Gamma^{-1}\left(z_{1}\right) \backslash\{y\}$ is uniformly distributed over all $(d-1)$-element subsets of $Y \backslash\{y\}$. Another way to interpret this identity is by ordering the edges coming in $z_{1}$. Without loss of generality we can assume that the first edge is $y z_{1}$. The probability the second edge coming in $z_{1}$ does not originate from $S$ is $1-s /(n-1)$. The probability the third edge coming in $z_{1}$ does not originate from $S$, given that the second does not, is $1-s /(n-2)$ and so on.

For the inductive step let us write $T^{\prime}=\left\{z_{1}, \ldots, z_{t-1}\right\}$. As

$$
\operatorname{Pr}\left(\Gamma^{-1}(T) \cap S=\emptyset\right)=\operatorname{Pr}\left(\Gamma^{-1}\left(T^{\prime}\right) \cap S=\emptyset\right) \operatorname{Pr}\left(\Gamma^{-1}\left(z_{t}\right) \cap S=\emptyset \mid \Gamma^{-1}\left(T^{\prime}\right) \cap S=\emptyset\right),
$$

it is enough for our purpose to establish that

$$
\begin{aligned}
\operatorname{Pr}\left(\Gamma^{-1}\left(z_{t}\right) \cap S=\emptyset \mid \Gamma^{-1}\left(T^{\prime}\right) \cap S=\emptyset\right) & \leqslant \operatorname{Pr}\left(\Gamma^{-1}\left(z_{t}\right) \cap S=\emptyset\right) \\
& =\operatorname{Pr}\left(\Gamma^{-1}\left(z_{1}\right) \cap S=\emptyset\right) .
\end{aligned}
$$

The last equality follows from the symmetry properties of biregular bipartite graphs. The remainder of the proof is dedicated to proving (2). The strategy is to order the edges ending in $z_{t}$ and successively estimate the probability that each does not originate from $S$. This will be done in a number of lemmata.

We need to keep track of the first $j$ edges ending in $z_{t}$. To achieve this we denote by $y_{1}, \ldots, y_{d}$ the elements of $\Gamma^{-1}\left(z_{t}\right)$, where $y=y_{1}$, and, for $1 \leqslant j \leqslant d$, we set $F_{j}=$ $\left\{y_{1}, \ldots, y_{j}\right\}$.

The key is to prove the following intuitively clear observation. Suppose that $\Gamma^{-1}\left(T^{\prime}\right)$ and $F_{j}$ are disjoint from $S$. Then for any $u \in S$ and any $v \in Y \backslash F_{j}$ the probability that $y_{j+1}=u$ is no smaller than the probability that $y_{j+1}=v$. We prove the statement in a number of steps. Initially we condition on $F_{j}$ and $\Gamma^{-1}\left(T^{\prime}\right)$.

Lemma 14. Let $1 \leqslant j \leqslant d-1$ be an integer and $u \in S$. Suppose $J \subseteq Y \backslash S$ is a set of size $j$ that contains $y, W \subset Y \backslash S$ is another subset of $Y$ that is disjoint from $S$ and $v \notin W \cup J$. Then

$$
\operatorname{Pr}\left(y_{j+1}=v \mid F_{j}=J \wedge \Gamma^{-1}\left(T^{\prime}\right)=W\right)=\operatorname{Pr}\left(y_{j+1}=u \mid F_{j}=J \wedge \Gamma^{-1}\left(T^{\prime}\right)=W\right) .
$$

Proof. The statement follows from the symmetry properties of random biregular bipartite graphs: interchanging $u$ and $v$ does not affect the events $\left\{\Gamma^{-1}\left(T^{\prime}\right)=W\right\}$ nor $\left\{F_{j}=J\right\}$.

Lemma 15. Let $1 \leqslant j \leqslant d-1$ be an integer and $u \in S$. Suppose $J \subseteq Y \backslash S$ is a set of size $j$ that contains $y, W \subset Y \backslash S$ is another subset of $Y$ that is disjoint from $S$ and $v \in W \backslash J$. Then

$$
\operatorname{Pr}\left(y_{j+1}=v \mid F_{j}=J \wedge \Gamma^{-1}\left(T^{\prime}\right)=W\right) \leqslant \operatorname{Pr}\left(y_{j+1}=u \mid F_{j}=J \wedge \Gamma^{-1}\left(T^{\prime}\right)=W\right) .
$$

Proof. We first observe that, say,

$$
\operatorname{Pr}\left(y_{j+1}=v \mid F_{j}=J \wedge \Gamma^{-1}\left(T^{\prime}\right)=W\right)=\frac{\operatorname{Pr}\left(v \in \Gamma^{-1}\left(z_{t}\right) \mid F_{j}=J \wedge \Gamma^{-1}\left(T^{\prime}\right)=W\right)}{d-j}
$$


since $v$ could be any of the $d-j$ remaining vertices in $\Gamma^{-1}\left(z_{t}\right) \backslash J$ with uniform probability. So the statement of the lemma is equivalent to

$$
\operatorname{Pr}\left(v \in \Gamma^{-1}\left(z_{t}\right) \mid F_{j}=J \wedge \Gamma^{-1}\left(T^{\prime}\right)=W\right) \leqslant \operatorname{Pr}\left(u \in \Gamma^{-1}\left(z_{t}\right) \mid F_{j}=J \wedge \Gamma^{-1}\left(T^{\prime}\right)=W\right) .
$$

Subtracting the probability $\operatorname{Pr}\left(\{u, v\} \subseteq \Gamma^{-1}\left(z_{t}\right) \mid F_{j}=J \wedge \Gamma^{-1}\left(T^{\prime}\right)=W\right)$ from both sides of the inequality leaves us with having to prove that

$$
\operatorname{Pr}\left(v \in \Gamma^{-1}\left(z_{t}\right) \wedge u \notin \Gamma^{-1}\left(z_{t}\right) \mid F_{j}=J \wedge \Gamma^{-1}\left(T^{\prime}\right)=W\right)
$$

is at most

$$
\operatorname{Pr}\left(u \in \Gamma^{-1}\left(z_{t}\right) \wedge v \notin \Gamma^{-1}\left(z_{t}\right) \mid F_{j}=J \wedge \Gamma^{-1}\left(T^{\prime}\right)=W\right) .
$$

To this end we define two families of graphs:

$$
\mathcal{G}_{v}=\left\{G \in \mathcal{G}(k, n, d): v \in \Gamma^{-1}\left(z_{t}\right) \wedge u \notin \Gamma^{-1}\left(z_{t}\right) \wedge F_{j}=J \wedge \Gamma^{-1}\left(T^{\prime}\right)=W\right\}
$$

and

$$
\mathcal{G}_{u}=\left\{G \in \mathcal{G}(k, n, d): u \in \Gamma^{-1}\left(z_{t}\right) \wedge v \notin \Gamma^{-1}\left(z_{t}\right) \wedge F_{j}=J \wedge \Gamma^{-1}\left(T^{\prime}\right)=W\right\}
$$

and we establish that $\left|\mathcal{G}_{v}\right| \leqslant\left|\mathcal{G}_{u}\right|$.

For this purpose it is advantageous to know the size of the intersection $\Gamma(u) \cap \Gamma(v)$. In $\mathcal{G}_{v}$ the intersection is at most $k d-2$ as both $z_{t}$ and an element of $T^{\prime}$ lie in $\Gamma(v) \backslash \Gamma(u)$. In $\mathcal{G}_{u}$ the intersection is at most $k d-1$ as $z_{t} \in \Gamma(u) \backslash \Gamma(v)$. For $0 \leqslant i \leqslant k d-2$ we define new families of graphs

$\mathcal{G}_{v, i}=\left\{G \in \mathcal{G}(k, n, d): v \in \Gamma^{-1}\left(z_{t}\right) \wedge u \notin \Gamma^{-1}\left(z_{t}\right) \wedge|\Gamma(u) \cap \Gamma(v)|=i \wedge F_{j}=J \wedge \Gamma^{-1}\left(T^{\prime}\right)=W\right\}$

and

$\mathcal{G}_{u, i}=\left\{G \in \mathcal{G}(k, n, d): u \in \Gamma^{-1}\left(z_{t}\right) \wedge v \notin \Gamma^{-1}\left(z_{t}\right) \wedge|\Gamma(u) \cap \Gamma(v)|=i \wedge F_{j}=J \wedge \Gamma^{-1}\left(T^{\prime}\right)=W\right\}$.

To finish the proof it is enough to show that for all $0 \leqslant i \leqslant k d-2$ we have $\left|\mathcal{G}_{v, i}\right| \leqslant\left|\mathcal{G}_{u, i}\right|$. We establish this by counting in two ways $N_{i}$, the number of switchings between $\mathcal{G}_{v, i}$ and $\mathcal{G}_{u, i}$. I.e. we double count the number of pairs $\left(G_{v}, G_{u}\right) \in \mathcal{G}_{v, i} \times \mathcal{G}_{u, i}$ where $G_{u}$ is a switching of $G_{v}$ or equivalently $G_{v}$ is a switching of $G_{u}$.

We pick $G_{v} \in \mathcal{G}_{v, i}$ and let $z \in \Gamma(u) \backslash \Gamma(v)$. Applying the $\left\{v z_{t}, u z\right\}$-switching to $G_{v}$ gives a graph $G_{u} \in \mathcal{G}_{u, i}$, as the switching does not affect neither $|\Gamma(u) \cap \Gamma(v)|$ nor the event $\left\{F_{j}=J \wedge \Gamma^{-1}\left(T^{\prime}\right)=W\right\}$, and disconnects $v$ from $z_{t}$ by connecting it to $u$ (see Figure 2 ). There are $k d-i$ vertices in $\Gamma(u) \backslash \Gamma(v)$ and so

$$
N_{i}=(k d-i)\left|\mathcal{G}_{v, i}\right| \text {. }
$$

Next we pick $G_{u} \in \mathcal{G}_{u, i}$ and let $z \in \Gamma(v) \backslash\left(\Gamma(u) \cup T^{\prime}\right)$. Just like above, applying the $\left\{u z_{t}, v z\right\}$-switching to $G_{u}$ gives a graph $G_{v} \in \mathcal{G}_{v, i}$. This time however there are at most $(k d-1-i)$ vertices in $\Gamma(v) \backslash\left(\Gamma(u) \cup T^{\prime}\right)$, as $v \in \Gamma^{-1}\left(T^{\prime}\right)$. Thus,

$$
N_{i} \leqslant(k d-i-1)\left|\mathcal{G}_{u, i}\right|
$$



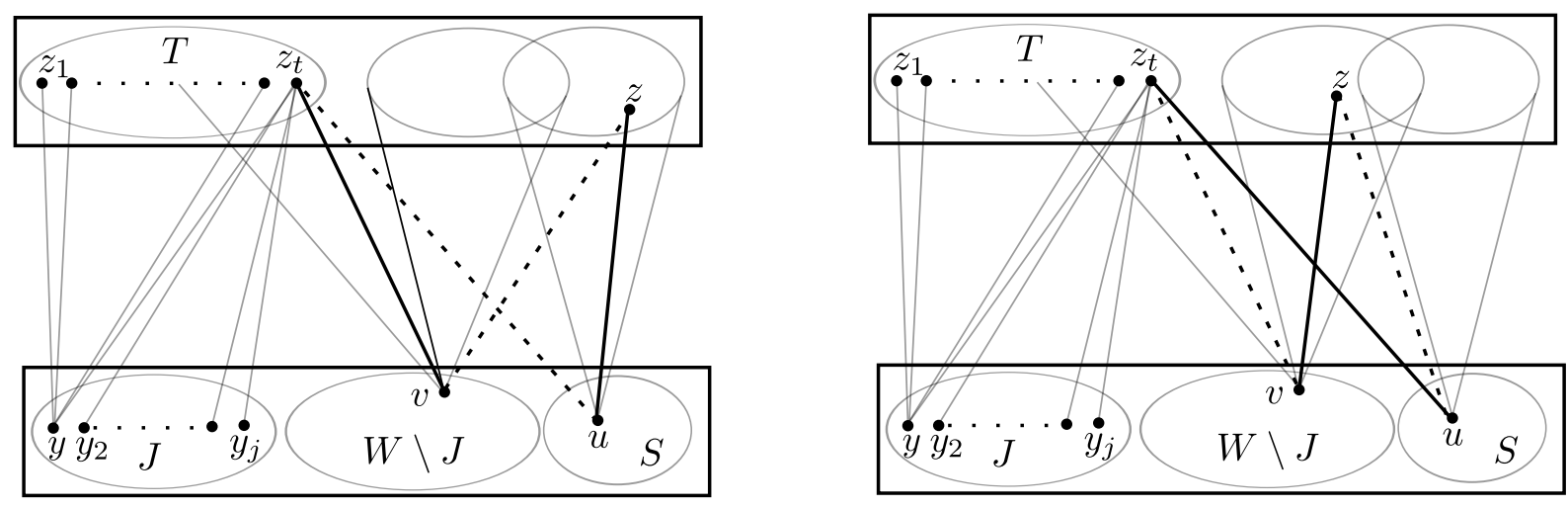

Figure 2: A graph $G_{v} \in \mathcal{G}_{v}$ and its switching $G_{u} \in \mathcal{G}_{u}$. Solid lines represent edges and dashed lines missing edges.

Comparing the lower and upper bounds for $N_{i}$ gives $\left|\mathcal{G}_{v, i}\right| \leqslant\left|\mathcal{G}_{u, i}\right|$, for $0 \leqslant i \leqslant k d-2$, and so

$$
\left|\mathcal{G}_{v}\right|=\sum_{i=0}^{k d-2}\left|\mathcal{G}_{v, i}\right| \leqslant \sum_{i=0}^{k d-2}\left|\mathcal{G}_{u, i}\right| \leqslant\left|\mathcal{G}_{u}\right|
$$

as required.

We resume the proof of Proposition 13 by noting that

$$
1=\sum_{v \in Y \backslash J} \operatorname{Pr}\left(y_{j+1}=v \mid F_{j}=J \wedge \Gamma^{-1}\left(T^{\prime}\right)=W\right),
$$

where $J$ and $W$ are taken to be subsets of $Y \backslash S$ as in the statement of the preceding lemma. The two preceding lemmata above imply that

$$
\operatorname{Pr}\left(y_{j+1}=v \mid F_{j}=J \wedge \Gamma^{-1}\left(T^{\prime}\right)=W\right) \leqslant \operatorname{Pr}\left(y_{j+1}=u \mid F_{j}=J \wedge \Gamma^{-1}\left(T^{\prime}\right)=W\right),
$$

for any $v \in Y \backslash J$ and some fixed $u \in S$. Substituting in the identity above and using the symmetry of the vertices in $S$ leads to

$$
\begin{aligned}
1 & \leqslant(n-j) \operatorname{Pr}\left(y_{j+1}=u \mid F_{j}=J \wedge \Gamma^{-1}\left(T^{\prime}\right)=W\right) \\
& =(n-j) \frac{\operatorname{Pr}\left(y_{j+1} \in S \mid F_{j}=J \wedge \Gamma^{-1}\left(T^{\prime}\right)=W\right)}{s},
\end{aligned}
$$

which in turn implies

$$
\begin{aligned}
\operatorname{Pr}\left(y_{j+1} \notin S \mid F_{j}=J \wedge \Gamma^{-1}\left(T^{\prime}\right)=W\right) & =1-\operatorname{Pr}\left(y_{j+1} \in S \mid F_{j}=J \wedge \Gamma^{-1}\left(T^{\prime}\right)=W\right) \\
& \leqslant 1-\frac{s}{n-j} .
\end{aligned}
$$

We take advantage of the fact that the upper bound is independent of $J$ and $W$ to deduce an upper bound for the probability $\operatorname{Pr}\left(y_{j+1} \notin S \mid\left(F_{j} \cup \Gamma^{-1}\left(T^{\prime}\right)\right) \cap S=\emptyset\right)$. 
Corollary 16. Let $1 \leqslant j \leqslant d-1$. Then $\operatorname{Pr}\left(y_{j+1} \notin S \mid\left(F_{j} \cup \Gamma^{-1}\left(T^{\prime}\right)\right) \cap S=\emptyset\right) \leqslant$ $1-s /(n-j)$.

Proof. The probability

$$
\operatorname{Pr}\left(y_{j+1} \notin S \mid\left(F_{j} \cup \Gamma^{-1}\left(T^{\prime}\right)\right) \cap S=\emptyset\right)
$$

equals

$\sum_{J, W} \operatorname{Pr}\left(y_{j+1} \notin S \mid F_{j}=J \wedge \Gamma^{-1}\left(T^{\prime}\right)=W\right) \operatorname{Pr}\left(F_{j}=J \wedge \Gamma^{-1}\left(T^{\prime}\right)=W \mid\left(F_{j} \cup \Gamma^{-1}\left(T^{\prime}\right)\right) \cap S=\emptyset\right)$,

where the sums are over all $j$-element subsets $J$ of $Y \backslash S$ and all subsets $W \subset Y \backslash S$. This in turn is at most

$$
\left(1-\frac{s}{n-j}\right) \sum_{J, W} \operatorname{Pr}\left(F_{j}=J \wedge \Gamma^{-1}\left(T^{\prime}\right)=W \mid\left(F_{j} \cup \Gamma^{-1}\left(T^{\prime}\right)\right) \cap S=\emptyset\right)=1-\frac{s}{n-j} .
$$

We can finally deduce (2). Recall that $\Gamma^{-1}\left(z_{t}\right)=\left\{y_{1}, \ldots, y_{d}\right\}$, where $y_{1}=y$.

$$
\begin{aligned}
\operatorname{Pr}\left(\Gamma^{-1}\left(z_{t}\right) \cap S=\emptyset \mid \Gamma^{-1}\left(T^{\prime}\right) \cap S=\emptyset\right) & =\prod_{j=1}^{d-1} \operatorname{Pr}\left(y_{j+1} \notin S \mid\left(F_{j} \cup \Gamma^{-1}\left(T^{\prime}\right)\right) \cap S=\emptyset\right) \\
& \leqslant \prod_{j=1}^{d-1}\left(1-\frac{s}{n-j}\right) \\
& =\operatorname{Pr}\left(\Gamma^{-1}\left(z_{1}\right) \cap S=\emptyset\right) .
\end{aligned}
$$

Equation (1) was used for the last equality. This finishes the inductive step and concludes the proof of Proposition 13.

A special case that will be of particular importance in the next section is when $S=$ $A \backslash\{y\}$ and $T$ is a singleton. For this case we would like to have not only an upper bound, but also an asymptotic expression for the probability that there are no edges between the two sets.

Lemma 17. Let $Y, A, Z, G$ and $y$ be like in the statement of Theorem 4. Suppose that $z_{1} \in \Gamma(y)$. Then

$$
\operatorname{Pr}\left(\Gamma^{-1}\left(z_{1}\right) \cap A=\{y\}\right)=(1+o(1)) \exp \left(-\frac{k d^{2}}{n}\right)
$$

provided only that $d=o\left(n^{2 / 3}\right)$. 
Proof. Recall that $|A|=k d$. Setting $T=\left\{z_{1}\right\}$ and $S=A \backslash\{y\}$ in Proposition 13 gives

$$
\operatorname{Pr}\left(\Gamma^{-1}\left(z_{1}\right) \cap A=\{y\}\right) \leqslant(1+o(1)) \exp \left(-\frac{k d^{2}}{n}\right) .
$$

To get a lower bound we first note that Proposition 13 gives

$$
\begin{aligned}
\operatorname{Pr}\left(\Gamma^{-1}\left(z_{1}\right) \cap A=\{y\}\right) & =\left(1-\frac{k d-1}{n-1}\right)\left(1-\frac{k d-1}{n-2}\right) \ldots\left(1-\frac{k d-1}{n-d+1}\right) \\
& \geqslant\left(1-\frac{k d-1}{n-d}\right)^{d-1} .
\end{aligned}
$$

We make use of the inequality $(1-x) \geqslant\left(1+O\left(x^{2}\right)\right) \exp \left(-x-x^{2}\right)$ which holds when $x \rightarrow 0$. When $d=o\left(n^{2 / 3}\right)$ the ratio $\frac{(k d-1)^{2}(d-1)}{(n-d)^{2}}=o(1)$ and so

$$
\begin{aligned}
\operatorname{Pr}\left(\Gamma^{-1}\left(z_{1}\right) \cap A=\{y\}\right) & \geqslant\left(1+O\left(\frac{(k d-1)^{2}}{(n-d)^{2}}\right)\right)^{d-1} \exp \left(-\frac{k d-1}{n-d}-\frac{(k d-1)^{2}}{(n-d)^{2}}\right)^{d-1} \\
& =(1+o(1)) \exp \left(-(d-1)\left(\frac{(k d-1)}{n}+O\left(\frac{k d^{2}}{n^{2}}\right)\right)\right) \\
& =(1+o(1)) \exp \left(-\frac{k d^{2}}{n}\right) .
\end{aligned}
$$

\section{Proof of Theorem 4}

When $d=o(\sqrt{n})$ it is straightforward to show there is no perfect matching in $H$ whp. Take $y^{\prime} \in A \backslash\{y\}$. By Lemma 11 we know that the expected value $\mathbb{E}\left(\left|\Gamma(y) \cap \Gamma\left(y^{\prime}\right)\right|\right)=o(1)$. Thus the probability $\operatorname{Pr}\left(\Gamma(y) \cap \Gamma\left(y^{\prime}\right)=\emptyset\right)=1-o(1)$ and consequently there is no perfect matching in $H$ with probability $1-o(1)$. For larger values of $d$ this simple argument does not work.

We follow Erdős and Rényi in relating the event of finding a perfect matching in $H$ to the event that the minimum degree of the induced subgraph is 1 . As $k$ is not necessarily 1 and $y$ lies in the bottom layer there is no symmetry between the top and bottom layers. To deal with this technical difficulty, we will to consider $\delta^{+}(H)$ and $\delta^{-}(H)$ separately. The proof of Theorem 4 is broken down to four steps.

The first is to obtain a qualitative description of the range of $d$ for which $\delta^{-}(H)=1$ $w h p$. Note that $\delta^{-}(H)$ cannot be zero since $y \in A$.

Lemma 18. Let $H$ be the graph introduced in Theorem 4 and

$$
c=\frac{k d^{2}}{n}-\log (k d) \text {. }
$$

Then 
(i) $\operatorname{Pr}\left(\delta^{-}(H)=1\right)=1-o(1)$ when $c \rightarrow-\infty$ or when $d$ is a constant.

(ii) $\operatorname{Pr}\left(\delta^{-}(H)>1\right)=1-o(1)$ when $c \rightarrow+\infty$.

Furthermore there is no perfect matching in $H$ whp when $c \rightarrow-\infty$.

Proof. We estimate the expectation and variance of the number of vertices $z \in \Gamma(y)$ that satisfy $\Gamma^{-1}(z) \cap A=\{y\}$. So for $z \in \Gamma(y)$ we define the event

$$
A_{z}^{-}=\left\{\Gamma^{-1}(z) \cap A=\{y\}\right\}
$$

and the random variable

$$
A^{-}=\sum_{z \in \Gamma(y)} 1_{A_{z}^{-}}
$$

The linearity of expectation and the symmetry of biregular bipartite graphs gives

$$
\mathbb{E}\left(A^{-}\right)=k d \operatorname{Pr}\left(A_{z_{1}}^{-}\right) \quad \text { for any } z_{1} \in \Gamma(y) .
$$

Setting $T=\left\{z_{1}\right\}$ and $S=A \backslash\{y\}$ in Proposition 13 yields

$$
\mathbb{E}\left(A^{-}\right) \leqslant(1+o(1)) k d \exp \left(-\frac{d(k d-1)}{n}\right)=O\left(k d \exp \left(-\frac{k d^{2}}{n}\right)\right)=O\left(e^{-c}\right) .
$$

When $c \rightarrow+\infty$ the expectation is $\mathbb{E}\left(A^{-}\right)=o(1)$ and so $\operatorname{Pr}\left(A^{-}>0\right) \leqslant \mathbb{E}\left(A^{-}\right)=o(1)$ and conclusion $(i i)$ follows.

When $c \leqslant 0$ we certainly have that $d=o\left(n^{2 / 3}\right)$ and so Lemma 17 gives

$$
\mathbb{E}\left(A^{-}\right)=\sum_{z \in \Gamma(y)} \operatorname{Pr}\left(\Gamma^{-1}(z) \cap A=\{y\}\right)=(1+o(1)) k d \exp \left(-\frac{k d^{2}}{n}\right)=(1+o(1)) e^{-c} .
$$

Assuming furthermore that $c \rightarrow-\infty$ gives $\mathbb{E}\left(A^{-}\right) \rightarrow+\infty$. To be able to say something about the probability $\operatorname{Pr}\left(A^{-}>0\right)$ we need to control the variance of $A^{-}$. Then,

$$
\begin{aligned}
\operatorname{Var}\left(A^{-}\right) & =\mathbb{E}\left(\left(A^{-}\right)^{2}\right)-\left(\mathbb{E}\left(A^{-}\right)\right)^{2} \\
& =\sum_{z \in \Gamma(y)} \sum_{z^{\prime} \in \Gamma(y)} \operatorname{Pr}\left(A_{z}^{-} \wedge A_{z^{\prime}}^{-}\right)-\left(\sum_{z \in \Gamma(y)} \operatorname{Pr}\left(A_{z}^{-}\right)\right)^{2} \\
& =\sum_{z \in \Gamma(y)} \sum_{z^{\prime} \neq z} \operatorname{Pr}\left(A_{z}^{-} \wedge A_{z^{\prime}}^{-}\right)+\sum_{z \in \Gamma(y)} \operatorname{Pr}\left(A_{z}^{-}\right)-\sum_{z \in \Gamma(y)} \sum_{z^{\prime} \in \Gamma(y)} \operatorname{Pr}\left(A_{z}^{-}\right)^{2} .
\end{aligned}
$$

Setting $T=\left\{z, z^{\prime}\right\}$ and $S=A \backslash\{y\}$ in Proposition 13 gives $\operatorname{Pr}\left(A_{z}^{-} \wedge A_{z^{\prime}}^{-}\right) \leqslant \operatorname{Pr}\left(A_{z}^{-}\right)^{2}$. Consequently

$$
\operatorname{Var}\left(A^{-}\right) \leqslant \sum_{z \in \Gamma(y)} \sum_{z^{\prime} \neq z} \operatorname{Pr}\left(A_{z}^{-}\right)^{2}+\sum_{z \in \Gamma(y)} \operatorname{Pr}\left(A_{z}^{-}\right)-\sum_{z \in \Gamma(y)} \sum_{z^{\prime} \in \Gamma(y)} \operatorname{Pr}\left(A_{z}^{-}\right)^{2} .
$$


Here we used the fact mentioned above that $\operatorname{Pr}\left(A_{z}^{-}\right)$is independent of $z$ and hence equals $\operatorname{Pr}\left(A_{z_{0}}^{-}\right)$for any $z_{0} \in \Gamma(y)$. The right hand side therefore becomes

$$
\begin{aligned}
|\Gamma(y)||\Gamma(y)-1| \operatorname{Pr}\left(A_{z_{0}}^{-}\right)^{2}+|\Gamma(y)| \operatorname{Pr}\left(A_{z_{0}}^{-}\right)-|\Gamma(y)|^{2} \operatorname{Pr}\left(A_{z_{0}}^{-}\right)^{2} & =|\Gamma(y)| \operatorname{Pr}\left(A_{z_{0}}^{-}\right)\left(1-\operatorname{Pr}\left(A_{z_{0}}^{-}\right)\right) \\
& =\sum_{z \in \Gamma(y)} \operatorname{Pr}\left(A_{z}^{-}\right)\left(1-\operatorname{Pr}\left(A_{z}^{-}\right)\right) \\
& \leqslant \sum_{z \in \Gamma(y)} \operatorname{Pr}\left(A_{z}^{-}\right)=\mathbb{E}\left(A^{-}\right) .
\end{aligned}
$$

So $\operatorname{Var}\left(A^{-}\right) \leqslant \mathbb{E}\left(A^{-}\right)$. We can now finish off the proof of the Proposition 18 by applying Chebyshev's inequality.

Lemma 19 (Chebyshev's inequality). Let $X$ be a non-negative random variable with expected value $\mu$ and non-zero variance $\sigma^{2}$. Then for any $x \in \mathbb{R}^{+}$

$$
\operatorname{Pr}(|X-\mu| \geqslant x \sigma) \leqslant \frac{1}{x^{2}} .
$$

Applying the inequality to $A^{-}$gives

$$
\operatorname{Pr}\left(\delta^{-}(H)>1\right)=\operatorname{Pr}\left(A^{-}=0\right) \leqslant \operatorname{Pr}\left(\left|A^{-}-\mathbb{E}\left(A^{-}\right)\right| \geqslant \mathbb{E}\left(A^{-}\right)\right) \leqslant \frac{1}{\mathbb{E}\left(A^{-}\right)}=o(1)
$$

when $c \rightarrow-\infty$, implying conclusion $(i)$.

For the final conclusion we observe that there can be no perfect matching in $H$ when $A^{-} \geqslant 2$ as there would then exist two vertices in $\Gamma(y)$ that are only joined to $y$. A second application of Chebyshev's inequality gives that $\operatorname{Pr}\left(A^{-} \geqslant 2\right)=1-o(1)$ when $c \rightarrow-\infty$.

We have proved the first statement in Theorem 4. The second statement is trickier.

The second step in the proof of Theorem 4 is to show that $\delta^{+}(H)>0 w h p$ when $c \rightarrow+\infty$.

Lemma 20. Let $H$ be the graph introduced in Theorem 4 and

$$
c=\frac{k d^{2}}{n}-\log (k d)
$$

Then

$$
\operatorname{Pr}\left(\delta^{+}(H)=0\right)=o(1)
$$

when $c \rightarrow+\infty$.

Proof. We estimate the expected number of vertices $y^{\prime} \in A \backslash\{y\}$ that satisfy $\Gamma\left(y^{\prime}\right) \cap \Gamma(y)=$ $\emptyset$. So for $y^{\prime} \in A \backslash\{y\}$ we define the event

$$
A_{y^{\prime}}^{+}=\left\{\Gamma\left(y^{\prime}\right) \cap \Gamma(y)=\emptyset\right\} ;
$$


and the random variable

$$
A^{+}=\sum_{y^{\prime} \in A \backslash\{y\}} 1_{A_{y^{\prime}}^{+}}
$$

The linearity of expectation and the symmetry of biregular bipartite graphs gives

$$
\mathbb{E}\left(A^{+}\right)=(k d-1) \operatorname{Pr}\left(A_{y^{\prime}}^{+}\right) \quad \text { for any } y^{\prime} \in A \backslash\{y\} .
$$

Setting $S=\left\{y^{\prime}\right\}$ and $T=\Gamma(y)$ in Proposition 13 yields

$$
\mathbb{E}\left(A^{+}\right)=O\left(k d \exp \left(-\frac{k d^{2}}{n}\right)\right)=O\left(e^{-c}\right) .
$$

When $c \rightarrow+\infty$ the expectation $\mathbb{E}\left(A^{+}\right)=o(1)$ and so the probability $\operatorname{Pr}\left(\delta^{+}(H)=0\right)=$ $\operatorname{Pr}\left(A^{+}>0\right)=o(1)$.

To prove the existence of a perfect matching in $H$ we will rely on the FrobeniusKönig theorem (see e.g. [14, Theorem 1.7.1]), which is equivalent to the well known Hall's theorem.

Lemma 21 (The Frobenius-König theorem). Let $A$ and $B$ be two sets of equal size. Suppose that $H$ is a bipartite graph with vertex set $(A, B)$. Then $H$ has no perfect matching if and only if there are non-empty sets $S \subseteq A$ and $T \subseteq B$ such that $|S|+|T|=|A|+1$ and $\Gamma(S) \cap T=\emptyset$.

For any $S \subseteq A$ and $T \subseteq B$, a pair $(S, T)$ is called problematic if $|S|+|T|=|A|+1$ and $\Gamma(S) \cap T=\emptyset$. We show that the probability that a problematic pair $(S, T)$ exists in $H$ is $o(1)$ when $c \rightarrow+\infty$. For technical reasons we need to distinguish between two ranges for $c$. The third step in the proof of Theorem 4 is to deal with the case when $c$ is larger than a constant multiple of $\log (k d)$.

Proposition 22. Let $H$ be the graph introduced in Theorem 4 and

$$
c=\frac{k d^{2}}{n}-\log (k d) .
$$

Suppose that $c \geqslant 5 \log (k d)$. Then

$$
\operatorname{Pr}(\text { There is no perfect matching in } H)=O\left(k^{2} d^{2} \exp \left(-\frac{k d^{2}}{2 n}\right)\right) \text {. }
$$

In particular there is a perfect matching in $H$ whp.

Proof. It follows by Lemma 21 that no perfect matching exists in $H$ if and only if there are non-empty sets $S \subseteq A \backslash\{y\}$ and $T \subseteq \Gamma(y)$ such that $|S|+|T|=k d+1$ and $\Gamma(S) \cap T=\emptyset$. So we want to bound from above the probability a problematic pair of sets $(S, T)$ exists. 
For a pair of fixed sets $(S, T)$ where $S \subseteq A \backslash\{y\}, T \subseteq \Gamma(y),|S|=j$ and $|T|=k d+1-j$, Proposition 13 gives

$$
\operatorname{Pr}(\Gamma(S) \cap T=\emptyset) \leqslant \exp \left(-\frac{d|S||T|}{n}\right)=\exp \left(-\frac{d j(k d+1-j)}{n}\right) .
$$

For a given $j$ there are at most $\left(\begin{array}{c}k d-1 \\ j\end{array}\right)\left(\begin{array}{c}k d \\ k d+1-j\end{array}\right) \leqslant\left(\begin{array}{c}k d \\ j\end{array}\right)\left(\begin{array}{c}k d \\ j-1\end{array}\right)$ possible problematic pairs of sets $(S, T)$. Applying a union bound gives

$\operatorname{Pr}($ There is no perfect matching in $H)=O\left(\sum_{j=1}^{k d}\left(\begin{array}{c}k d \\ j\end{array}\right)\left(\begin{array}{c}k d \\ j-1\end{array}\right) \exp \left(-\frac{j(k d-j+1) d}{n}\right)\right)$.

Changing $j$ to $k d-j+1$ does not affect the summand, so

$\operatorname{Pr}($ There is no perfect matching in $H)=O\left(\sum_{j=1}^{k d / 2}\left(\begin{array}{c}k d \\ j\end{array}\right)\left(\begin{array}{c}k d \\ j-1\end{array}\right) \exp \left(-\frac{j(k d-j+1) d}{n}\right)\right)$

$$
\begin{aligned}
& =O\left(\sum_{1 \leqslant j \leqslant k d / 2}\left(\begin{array}{c}
k d \\
j
\end{array}\right)^{2} \exp \left(-\frac{j(k d-j) d}{n}\right)\right) \\
& =O\left(\sum_{1 \leqslant j \leqslant k d / 2}\left(k^{2} d^{2} \exp \left(-\frac{(k d-j) d}{n}\right)\right)^{j}\right) \\
& =O\left(\sum_{j=1}^{\infty}\left(k^{2} d^{2} \exp \left(-\frac{k d^{2}}{2 n}\right)\right)^{j}\right) .
\end{aligned}
$$

The lower bound on $c$ implies that $k d \rightarrow+\infty$ when $n \rightarrow+\infty$ and that $k^{2} d^{2} \exp \left(-\frac{k d^{2}}{2 n}\right)=$ $O\left((k d)^{-1}\right)<1$. So

$\operatorname{Pr}($ There is no perfect matching in $H)=O\left(k^{2} d^{2} \exp \left(-\frac{k d^{2}}{2 n}\right)\right)=O\left((k d)^{-1}\right)=o(1)$.

The above argument does not work when we merely assume that $c \rightarrow+\infty$. The forth and final task in the proof of Theorem 4 is to adapt the argument provided by Erdös and Rényi in [7] to the induced subgraph $H$.

The key is to consider the minimum out and in degrees. Lemma 18 and Lemma 20 combined imply that $\operatorname{Pr}\left(\left\{\delta^{-}(H)=1 \vee \delta^{+}(H)=0\right\}\right)=o(1)$ when $c \rightarrow+\infty$. So $\operatorname{Pr}($ There is no perfect matching in $H)$

$=\operatorname{Pr}\left(\right.$ There is no perfect matching in $\left.H \wedge \delta^{-}(H)>1 \wedge \delta^{+}(H)>0\right)+$

$\operatorname{Pr}$ (There is no perfect matching in $\left.H \wedge\left\{\delta^{-}(H)=1 \vee \delta^{+}(H)=0\right\}\right)$

$\leqslant \operatorname{Pr}$ (There is no perfect matching in $\left.H \wedge \delta^{-}(H)>1 \wedge \delta^{+}(H)>0\right)+$

$\operatorname{Pr}\left(\delta^{-}(H)=1 \vee \delta^{+}(H)=0\right)$

$=\operatorname{Pr}\left(\right.$ There is no perfect matching in $\left.H \wedge \delta^{-}(H)>1 \wedge \delta^{+}(H)>0\right)+o(1)$. 
So we are only left with proving that $\operatorname{Pr}$ (There is no perfect matching in $H \wedge \delta^{-}(H)>$ $\left.1 \wedge \delta^{+}(H)>0\right)=o(1)$ when $c \rightarrow+\infty$ and $c \leqslant 5 \log (k d)$.

Proposition 23. Let $H$ be the graph introduced in Theorem 4 and

$$
c=\frac{k d^{2}}{n}-\log (k d) .
$$

Suppose that $0 \leqslant c \leqslant 5 \log (k d)$. Then

$\operatorname{Pr}\left(\right.$ There is no perfect matching in $\left.H \wedge \delta^{-}(H)>1 \wedge \delta^{+}(H)>0\right)=o(1)$.

Proof. We once again apply Lemma 21: no perfect matching exists in $H$ if there are non-empty sets $S \subseteq A \backslash\{y\}$ and $T \subseteq \Gamma(y)$ such that $|S|+|T|=k d+1$ and $\Gamma(S) \cap T=\emptyset$. We consider the cases $|S| \leqslant|T|$ and $|T|<|S|$ separately. Let us start with the former.

Note that $\delta^{+}(H)>0$ implies that if $S$ belongs to a problematic pair, then $|S|>1$. Suppose for a contradiction that $|S|=1$. Then $T=\Gamma(y)$ and, since $(S, T)$ is problematic, there must be no edges between $S$ and $T$ and hence $\delta^{+}(H)=0$.

The size of $S$ lies in the range $2 \leqslant|S| \leqslant(k d+1) / 2$ since $|S| \leqslant|T|$. The key is to only consider $S$-minimal problematic pairs $(S, T)$. This means that there exists no proper subset $S^{\prime} \subsetneq S \subseteq A$ and $T^{\prime} \subseteq \Gamma(y)$ with $\left|S^{\prime}\right|+\left|T^{\prime}\right|=k d+1$ such that $\left(S^{\prime}, T^{\prime}\right)$ is a problematic pair.

We crucially observe that every $w \in \Gamma(y) \backslash T$ must have at least two edges landing in $S$. Otherwise, picking a $w \in \Gamma(y) \backslash T$ and $s \in S$ such that $E(S,\{w\})=\{s w\}$ and replacing $(S, T)$ by $(S \backslash\{s\}, T \cup\{w\})$ gives another problematic pair, which contradicts the minimality of $S$.

Keeping all this in mind let us calculate the probability that such a problematic pair of sets $(S, T)$ exists. We fix $S \subseteq A \backslash\{y\}$ and $T \subseteq \Gamma(y)$ with $|S|+|T|=k d+1$ and let $j=|S|$. We have to bound from above the probability that there are no edges from $S$ to $T$ and that all the vertices in $\Gamma(y) \backslash T$ have at least two edges starting in $S$. To keep the notation simple let us write $\Gamma(y) \backslash T=\left\{w_{1}, \ldots, w_{j-1}\right\}$ and also name some events:

$$
E_{0}=\left\{\Gamma^{-1}(T) \cap S=\emptyset\right\}
$$

and for $1 \leqslant i \leqslant j-1$,

$$
E_{i}=\bigwedge_{\ell=1}^{i}\left\{\left|\Gamma^{-1}\left(w_{\ell}\right) \cap S\right| \geqslant 2\right\} .
$$

In this notation we have to bound

$$
\operatorname{Pr}\left(E_{0} \wedge E_{j-1}\right)=\operatorname{Pr}\left(E_{0}\right) \prod_{i=1}^{j-1} \operatorname{Pr}\left(E_{i} \mid E_{0} \wedge E_{i-1}\right) .
$$

Recall that Proposition 13 states that for a fixed pair of sets $(S, T)$ where $|S|=j$, the probability that $E_{0}$ occurs is bounded above by

$$
\operatorname{Pr}\left(E_{0}\right) \leqslant(1+o(1)) \exp \left(-\frac{d j(k d+1-j)}{n}\right) .
$$


So we are left to bound

$$
\begin{aligned}
\operatorname{Pr}\left(E_{i} \mid E_{0} \wedge E_{i-1}\right) & =\operatorname{Pr}\left(\left|\Gamma^{-1}\left(w_{i}\right) \cap S\right| \geqslant 2 \mid E_{0} \wedge E_{i-1}\right) \\
& \leqslant \sum_{s \neq s^{\prime} \in S} \operatorname{Pr}\left(\left\{s, s^{\prime}\right\} \subseteq \Gamma^{-1}\left(w_{i}\right) \mid E_{0} \wedge E_{i-1}\right) .
\end{aligned}
$$

Edges in $G$ are chosen with probability $d / n$. If they were chosen independently, then the right hand side would be $\left(\begin{array}{l}j \\ 2\end{array}\right)(d / n)^{2}$. We show that a similar upper bound holds for (6).

Lemma 24. Let $s$ and $s^{\prime}$ be two distinct vertices in $S$ and $1 \leqslant i \leqslant j-1$ be an integer. In the notation established above we have

$$
\operatorname{Pr}\left(\left\{s, s^{\prime}\right\} \subseteq \Gamma^{-1}\left(w_{i}\right) \mid E_{0} \wedge E_{i-1}\right) \leqslant\left(\frac{d}{n-d}\right)^{2} .
$$

Proof. As

$$
\operatorname{Pr}\left(\left\{s, s^{\prime}\right\} \subseteq \Gamma^{-1}\left(w_{i}\right) \mid E_{0} \wedge E_{i-1}\right)
$$

equals

$$
\operatorname{Pr}\left(s \in \Gamma^{-1}\left(w_{i}\right) \mid E_{0} \wedge E_{i-1}\right) \operatorname{Pr}\left(s^{\prime} \in \Gamma^{-1}\left(w_{i}\right) \mid E_{0} \wedge E_{i-1} \wedge s \in \Gamma^{-1}\left(w_{i}\right)\right),
$$

it is enough to prove that both probabilities appearing in the product above are at most $d /(n-d)$. Let us temporarily set $W_{i-1}=\left\{w_{1}, \ldots, w_{i-1}\right\}$.

To bound the first probability in (7) we observe that

$$
\begin{aligned}
k d & =\mathbb{E}\left(d^{+}(s) \mid E_{0} \wedge E_{i-1}\right) \\
& =\sum_{z \in Z \backslash T} \operatorname{Pr}\left(z \in \Gamma(s) \mid E_{0} \wedge E_{i-1}\right) \\
& \geqslant \sum_{z \in Z \backslash\left(T \cup W_{i-1}\right)} \operatorname{Pr}\left(z \in \Gamma(s) \mid E_{0} \wedge E_{i-1}\right) \\
& =(k n-k d+j-i) \operatorname{Pr}\left(w_{i} \in \Gamma(s) \mid E_{0} \wedge E_{i-1}\right) \\
& \geqslant(k n-k d) \operatorname{Pr}\left(s \in \Gamma^{-1}\left(w_{i}\right) \mid E_{0} \wedge E_{i-1}\right) .
\end{aligned}
$$

The third (and final) equality follows from the symmetry of random biregular biparite graphs.

For the second probability in (7) we proceed similarly

$$
\begin{aligned}
k d & =\mathbb{E}\left(d^{+}\left(s^{\prime}\right) \mid E_{0} \wedge E_{i-1} \wedge s \in \Gamma^{-1}\left(w_{i}\right)\right) \\
& \geqslant \sum_{z \in Z \backslash\left(T \cup W_{i-1}\right)} \operatorname{Pr}\left(z \in \Gamma\left(s^{\prime}\right) \mid E_{0} \wedge E_{i-1} \wedge s \in \Gamma^{-1}\left(w_{i}\right)\right) .
\end{aligned}
$$

In this case we can not deduce the desired bound from the symmetry of random biregular bipartite graphs since not all $z \in Z \backslash\left(T \cup W_{i-1}\right)$ have the same role in the graph. Instead we prove via switching that the probability appearing in the sum above is minimal when $z=w_{i}$. 
Claim.

$$
\operatorname{Pr}\left(w_{i} \in \Gamma\left(s^{\prime}\right) \mid E_{0} \wedge E_{i-1} \wedge s \in \Gamma^{-1}\left(w_{i}\right)\right) \leqslant \operatorname{Pr}\left(z \in \Gamma\left(s^{\prime}\right) \mid E_{0} \wedge E_{i-1} \wedge s \in \Gamma^{-1}\left(w_{i}\right)\right),
$$

for all $z \in Z \backslash\left(T \cup W_{i-1}\right)$.

Let us quickly deduce the required inequality for the second probability appearing in (7) before proving the claim:

$$
\begin{aligned}
k d & \geqslant(k n-k d+j-i) \operatorname{Pr}\left(w_{i} \in \Gamma\left(s^{\prime}\right) \mid E_{0} \wedge E_{i-1} \wedge s \in \Gamma^{-1}\left(w_{i}\right)\right) \\
& \geqslant(k n-k d) \operatorname{Pr}\left(s^{\prime} \in \Gamma^{-1}\left(w_{i}\right) \mid E_{0} \wedge E_{i-1} \wedge s \in \Gamma^{-1}\left(w_{i}\right)\right) .
\end{aligned}
$$

Proof of the claim. We only need to prove the claim when $z \neq w_{i}$. Subtracting the probability

$$
\operatorname{Pr}\left(\left\{w_{i}, z\right\} \subseteq \Gamma\left(s^{\prime}\right) \mid E_{0} \wedge E_{i-1} \wedge s \in \Gamma^{-1}\left(w_{i}\right)\right)
$$

from both sides of the inequality we see that we have to prove that

$$
\operatorname{Pr}\left(w_{i} \in \Gamma\left(s^{\prime}\right) \wedge z \notin \Gamma\left(s^{\prime}\right) \mid E_{0} \wedge E_{i-1} \wedge s \in \Gamma^{-1}\left(w_{i}\right)\right)
$$

is at most

$$
\operatorname{Pr}\left(w_{i} \notin \Gamma\left(s^{\prime}\right) \wedge z \in \Gamma\left(s^{\prime}\right) \mid E_{0} \wedge E_{i-1} \wedge s \in \Gamma^{-1}\left(w_{i}\right)\right) .
$$

This is equivalent to proving that $\left|\mathcal{G}_{w_{i}}\right| \leqslant\left|\mathcal{G}_{z}\right|$ where

$$
\mathcal{G}_{w_{i}}=\left\{G \in \mathcal{G}(k, n, d): w_{i} \in \Gamma\left(s^{\prime}\right) \wedge z \notin \Gamma\left(s^{\prime}\right) \wedge E_{0} \wedge E_{i-1} \wedge s \in \Gamma^{-1}\left(w_{i}\right)\right\}
$$

and

$$
\mathcal{G}_{z}=\left\{G \in \mathcal{G}(k, n, d): z \in \Gamma\left(s^{\prime}\right) \wedge w_{i} \notin \Gamma\left(s^{\prime}\right) \wedge E_{0} \wedge E_{i-1} \wedge s \in \Gamma^{-1}\left(w_{i}\right)\right\} .
$$

Like in the proof of Lemma 15, we partition the two families of graphs in subfamilies according to the size of the intersection $\Gamma^{-1}\left(w_{i}\right) \cap \Gamma^{-1}(z)$. For any $0 \leqslant \ell \leqslant d-1$ we define $\mathcal{G}_{w_{i}, \ell}=\left\{G \in \mathcal{G}(k, n, d): w_{i} \in \Gamma\left(s^{\prime}\right) \wedge z \notin \Gamma\left(s^{\prime}\right) \wedge E_{0} \wedge E_{i-1} \wedge s \in \Gamma^{-1}\left(w_{i}\right) \wedge\left|\Gamma^{-1}\left(w_{i}\right) \cap \Gamma^{-1}(z)\right|=\ell\right\}$ and

$\mathcal{G}_{z, \ell}=\left\{G \in \mathcal{G}(k, n, d): z \in \Gamma\left(s^{\prime}\right) \wedge w_{i} \notin \Gamma\left(s^{\prime}\right) \wedge E_{0} \wedge E_{i-1} \wedge s \in \Gamma^{-1}\left(w_{i}\right) \wedge\left|\Gamma^{-1}\left(w_{i}\right) \cap \Gamma^{-1}(z)\right|=\ell\right\}$.

The parameter $\ell$ is at most $d-1$ in both $\mathcal{G}_{w_{i}}$ and $\mathcal{G}_{z}$ as $s^{\prime}$ lies in exactly one of the two sets $\Gamma^{-1}\left(w_{i}\right)$ and $\Gamma^{-1}(z)$. For $0 \leqslant \ell \leqslant d-1$ we count in two ways $N_{\ell}$, the number of switchings between $\mathcal{G}_{w_{i}, \ell}$ and $\mathcal{G}_{z, \ell}$. In other words we double count the number of ordered pairs $\left(G_{w_{i}}, G_{z}\right) \in \mathcal{G}_{w_{i}, \ell} \times \mathcal{G}_{z, \ell}$ such that $G_{w_{i}}$ is a switching of $G_{z}$ or equivalently that $G_{z}$ is a switching of $G_{w_{i}}$.

Take $G_{w_{i}} \in \mathcal{G}_{w_{i}, \ell}$ and $v \in \Gamma^{-1}(z) \backslash \Gamma^{-1}\left(w_{i}\right)$. Applying the $\left\{s^{\prime} w_{i}, v z\right\}$-switching to $G_{w_{i}}$ results in a member of $\mathcal{G}_{z, \ell}$ as the switching does not affect any of the events $E_{0}, E_{i-1},\{s \in$ $\left.\Gamma^{-1}\left(w_{i}\right)\right\}$ and $\left\{\left|\Gamma^{-1}\left(w_{i}\right) \cap \Gamma^{-1}(z)\right|=\ell\right\}$ (see Figure 3 ). There are $(d-\ell)$ such $v$ and so

$$
N_{\ell}=(d-\ell)\left|\mathcal{G}_{w_{i}, \ell}\right| .
$$



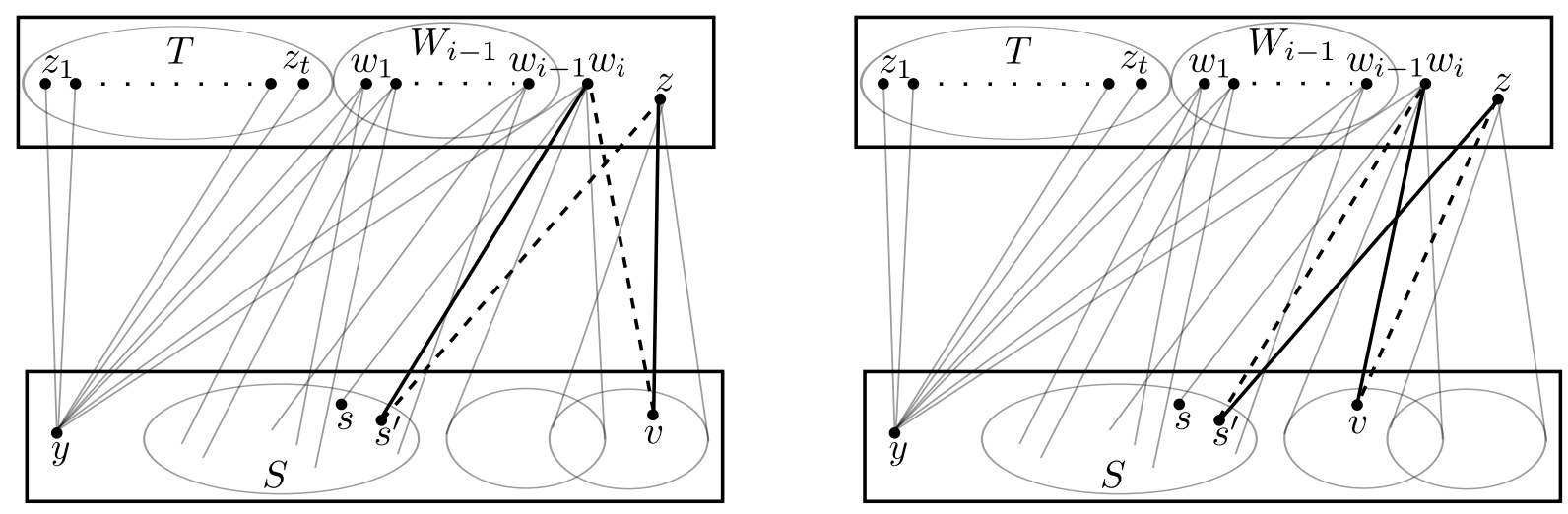

Figure 3: A graph $G_{w_{i}} \in \mathcal{G}_{w_{i}}$ and its switching $G_{z} \in \mathcal{G}_{z}$. Solid lines represent edges and dashed lines missing edges.

Now take $G_{z} \in \mathcal{G}_{z, \ell}$ and $v \in \Gamma^{-1}\left(w_{i}\right) \backslash\left(\Gamma^{-1}(z) \cup\{y, s\}\right)$. Applying the $\left\{s^{\prime} z, v w_{i}\right\}$-switching to $G_{z}$ results in a member of $\mathcal{G}_{w_{i}, \ell}$ as the switching does not affect any of the events $E_{0}, E_{i-1},\left\{s \in \Gamma^{-1}\left(w_{i}\right)\right\}$ and $\left\{\left|\Gamma^{-1}\left(w_{i}\right) \cap \Gamma^{-1}(z)\right|=\ell\right\}$. There are at most $(d-\ell)$ such $v$ and so

$$
N_{\ell} \leqslant(d-\ell)\left|\mathcal{G}_{z, \ell}\right| .
$$

Thus $\left|\mathcal{G}_{w_{i}, \ell}\right| \leqslant\left|\mathcal{G}_{z, \ell}\right|$ for all $0 \leqslant \ell \leqslant d-1$ and

$$
\left|\mathcal{G}_{w_{i}}\right|=\sum_{\ell=0}^{d-1}\left|\mathcal{G}_{w_{i}, \ell}\right| \leqslant \sum_{\ell=0}^{d-1}\left|\mathcal{G}_{z, \ell}\right|=\left|\mathcal{G}_{z}\right|
$$

This completes the proof of the lemma.

We resume the proof of Proposition 23. Inequality (6) becomes

$$
\operatorname{Pr}\left(E_{i} \mid E_{i-1} \wedge E_{0}\right) \leqslant\left(\begin{array}{l}
j \\
2
\end{array}\right)\left(\frac{d}{n-d}\right)^{2} .
$$

Substituting the above and the bound on $\operatorname{Pr}\left(E_{0}\right)$ from Proposition 13 in (5) gives

$$
\operatorname{Pr}\left(E_{0} \wedge E_{j-1}\right) \leqslant(1+o(1))\left(\begin{array}{l}
j \\
2
\end{array}\right)^{j-1}\left(\frac{d}{n-d}\right)^{2(j-1)} \exp \left(-\frac{\operatorname{dj}(k d+1-j)}{n}\right) .
$$

To bound the probability there is an $S$-minimal problematic pair with $|S| \leqslant|T|$ we apply a union bound. For fixed $j$ there are at most $\left(\begin{array}{c}k d \\ j\end{array}\right)\left(\begin{array}{c}k d \\ j-1\end{array}\right)$ ways to choose $(S, T)$ subject to $|S|=j$. Thus the probability that there is an $S$-minimal problematic pair with $|S| \leqslant|T|$ is

$$
O\left(\sum_{j=2}^{(k d+1) / 2}\left(\begin{array}{c}
k d \\
j
\end{array}\right)\left(\begin{array}{c}
k d \\
j-1
\end{array}\right)\left(\begin{array}{l}
j \\
2
\end{array}\right)^{j-1}\left(\frac{d}{n-d}\right)^{2(j-1)} \exp \left(-\frac{d j(k d+1-j)}{n}\right)\right)
$$


Applying the well known bound $\left(\begin{array}{l}a \\ b\end{array}\right) \leqslant\left(\frac{e a}{b}\right)^{b}$, using that $n-d \geqslant n / 2$ as $c=O(\log (k d))$ and writing $\ell=j-1$ we get that the probability that there is an $S$-minimal problematic pair with $|S| \leqslant|T|$ is

$$
O\left(\sum_{\ell=1}^{k d / 2} k d e^{-k d^{2} / n}\left(C \frac{k^{2} d^{4} e^{-d(k d-\ell) / n}}{n^{2}}\right)^{\ell}\right)
$$

for some large enough constant $C$.

The definition of $c$ gives that $e^{-k d^{2} / n}=e^{-c} / k d$. Observing that $k d-\ell \geqslant k d / 2$, the above sum is

$$
O\left(e^{-c} \sum_{\ell=1}^{k d / 2}\left(C\left(\frac{k d^{2}}{n}\right)^{2} \frac{e^{-c / 2}}{\sqrt{k d}}\right)^{\ell}\right)
$$

As $k d^{2} / n \leqslant 6 \log (k d)$ and $e^{-c} \leqslant 1$ we get that the probability a problematic pair exists with $|S| \leqslant|T|$ is

$$
O\left(\sum_{l=1}^{\infty}\left(C^{\prime} \frac{\log ^{2}(k d)}{\sqrt{k d}}\right)^{\ell}\right)=O\left(\frac{\log ^{2}(k d)}{\sqrt{k d}}\right)=o(1)
$$

where $C^{\prime}$ is another large enough constant.

The case when $|T|<|S|$ is similar. This time we chose $T$-minimal problematic pairs. The set $T$ cannot be a singleton as $\delta^{-}(H)>1$. The minimality of $|T|$ implies that for every $v \in A \backslash S$ there are at least two edges starting at $v$ and ending in $T$. The calculations needed are very similar to those given above and are omitted.

Let us quickly recap the proof of Theorem 4 .

Proof of Theorem 4. When $c \rightarrow-\infty$ Lemma 18 gives that there is no perfect matching in $H$ whp.

When $c \rightarrow+\infty$ Lemma 18 and Lemma 20 give that $\operatorname{Pr}\left(\delta^{-}(H)>1 \vee \delta^{+}(H)>0\right)=$ $1-o(1)$. Proposition 23 states that the probability there is no perfect matching and $\delta^{+}(H)>0$ or $\delta^{-}(H)>1$ is $o(1)$ provided that $c \leqslant 5 \log (k d)$. So there is a perfect matching in $H$ whp when $c \rightarrow+\infty$ and $c \leqslant 5 \log (k d)$. Finally Proposition 22 gives that there is a perfect matching in $H$ whp when $c \geqslant 5 \log (k d)$.

We conclude the section with some remarks on the probability of the events $A_{y^{\prime}}^{+}$defined in (4) at the beginning of the proof of Lemma 20 on p.15. Suppose for a moment that $G$ was chosen uniformly at random from the family of $d^{+}$-regular bipartite graphs. In other words $d^{+}$would be constant (and equal to $k d$ ), but no restriction on $d^{-}$would exist. Then the neighbourhoods of vertices in $Y$ would be chosen uniformly at random from all $(k d)$-element subsets of $Z$ and independently of each other. So the probability that $\Gamma(y) \cap \Gamma\left(y^{\prime}\right)=\emptyset$ would be equal to $\left(\begin{array}{c}k n-k d \\ k d\end{array}\right) /\left(\begin{array}{c}k n \\ k d\end{array}\right)$. 
The probability that $A_{y^{\prime}}^{+}$occurs increases in random biregular bipartite graphs as the event $\left\{\Gamma(y)=S_{1} \wedge \Gamma\left(y^{\prime}\right)=S_{2}\right\}$ is more likely when $S_{1} \cap S_{2}=\emptyset$ than when $S_{1} \cap S_{2} \neq \emptyset$. This can be proved via switching and is due to the fact that vertices in $\Gamma(y)$ have one of their $d$ incoming edges "taken up" by $y$. Thus the edges coming out of $y^{\prime}$ are more likely to land in $Z \backslash \Gamma(y)$. We do not give the details of the proof here as a lower bound on $\operatorname{Pr}\left(A_{y^{\prime}}^{+}\right)$is not necessary. We only state the lower bound and compare it with the upper bound coming from Proposition 13:

$$
\frac{\left(\begin{array}{c}
k n-k d \\
k d
\end{array}\right)}{\left(\begin{array}{c}
k n \\
k d
\end{array}\right)} \leqslant \operatorname{Pr}\left(A_{y^{\prime}}^{+}\right) \leqslant\left(\frac{\left(\begin{array}{c}
n-2 \\
d-1
\end{array}\right)}{\left(\begin{array}{c}
n-1 \\
d-1
\end{array}\right)}\right)^{k d} .
$$

For $d=o\left(n^{2 / 3}\right)$ both bounds are asymptotically equal to $\exp \left(-k d^{2} / n\right)$, which is easy to see using Stirling approximation to the binomial coefficients. This reinforces the idea that the dependence among small sets of edges in $G(k, n, d)$ is small.

\section{Remarks on Theorem 2}

We only outline the proof of Theorem 2 as it is very similar to that of Theorem 4 . The difference lies in the induced subgraph under consideration. In Theorem $2, H$ is defined to be $G[A, B]$ where $A \subseteq Y$ and $B \subseteq Z$ are sets of size $k d$. In Theorem $4, B$ is taken to be the neighbourhood of some $y \in A$. This complicates some parts of the proof and is why we opted to give the proof of Theorem 4 .

When $d=o(\sqrt{n})$ it is straightforward to show there is no perfect matching in $H$ whp. By Lemma 11 we know that for any $y \in A$ the expected value $\mathbb{E}(|\Gamma(y) \cap B|)=o(1)$. Thus the probability $\operatorname{Pr}(\Gamma(y) \cap B=\emptyset)=1-o(1)$ and consequently there is no perfect matching in $H$ whp.

The first step in dealing with larger values of $d$ is to prove a variation of Proposition 13. As $y$ no longer has a special role it is possible to bound the probability there are no edges from $S$ to $T$ by looking one by one at the vertices of $S$ or $T$. In Proposition 13 we only worked with the vertices in $T$.

Proposition 25. Let $Y, A, Z, B$ and $G$ be like in the statement of Theorem 2. Let $S \subseteq A$ and $T \subseteq B$.

Suppose that $z \in T$ and $|S|+d \leqslant n$. Then

$$
\begin{aligned}
\operatorname{Pr}(\Gamma(S) \cap T=\emptyset) & \leqslant \operatorname{Pr}\left(\Gamma^{-1}(z) \cap S=\emptyset\right)^{|T|} \\
& \leqslant\left(1-\frac{|S|}{n}\right)^{|T|}\left(1-\frac{|S|}{n-1}\right)^{|T|} \ldots\left(1-\frac{|S|}{n-d+1}\right)^{|T|} \\
& \leqslant(1+o(1)) \exp \left(-\frac{d|S||T|}{n}\right) .
\end{aligned}
$$


Suppose that $y \in S$ and $|T|+k d \leqslant k n$. Then

$$
\begin{aligned}
\operatorname{Pr}(\Gamma(S) \cap T=\emptyset) & \leqslant \operatorname{Pr}(\Gamma(y) \cap T=\emptyset)^{|S|} \\
& \leqslant\left(1-\frac{|T|}{k n}\right)^{|S|}\left(1-\frac{|T|}{k n-1}\right)^{|S|} \ldots\left(1-\frac{|T|}{k n-k d+1}\right)^{|S|} \\
& \leqslant(1+o(1)) \exp \left(-\frac{d|S||T|}{n}\right) .
\end{aligned}
$$

Sketch of proof. For $z \in Z$ the probability there are no edges from $S$ to $z$ equals

$$
\operatorname{Pr}\left(\Gamma^{-1}(z) \cap S=\emptyset\right)=\frac{\left(\begin{array}{c}
n-|S| \\
d
\end{array}\right)}{\left(\begin{array}{c}
n \\
d
\end{array}\right)}=\left(1-\frac{|S|}{n}\right)\left(1-\frac{|S|}{n-1}\right) \ldots\left(1-\frac{|S|}{n-d+1}\right)
$$

as $\Gamma^{-1}(z)$ is chosen uniformly at random from all $d$-element subsets of $Y \backslash S$.

Now let $T=\left\{z_{1}, \ldots, z_{t}\right\}$. It can be shown via a switching argument very similar to that in the proof of Proposition 13 that for $2 \leqslant i \leqslant t$

$$
\begin{aligned}
\operatorname{Pr}\left(\Gamma^{-1}\left(z_{i}\right) \cap S=\emptyset \mid \Gamma^{-1}\left(\left\{z_{1}, \ldots, z_{i-1}\right\}\right) \cap S=\emptyset\right) & \leqslant \operatorname{Pr}\left(\Gamma^{-1}\left(z_{i}\right) \cap S=\emptyset\right) \\
& =\operatorname{Pr}\left(\Gamma^{-1}(z) \cap S=\emptyset\right) .
\end{aligned}
$$

This leads to

$$
\operatorname{Pr}(\Gamma(S) \cap T=\emptyset) \leqslant \operatorname{Pr}\left(\Gamma^{-1}(z) \cap S=\emptyset\right)^{|T|} .
$$

A similar approach is applied for the second claim.

Next we prove a variation of Lemma 18 for the minimum degree of $H, \delta(H)=$ $\min \left\{\delta^{+}(H), \delta^{-}(H)\right\}$. We no longer need to distinguish between $\delta^{+}(H)$ and $\delta^{-}(H)$ since $B \subseteq Z$ is an arbitrary set.

Lemma 26. Let $H$ be the graph introduced in Theorem 2 and

$$
c=\frac{k d^{2}}{n}-\log (k d)
$$

Then

(i) $\delta(H)=0$ whp when $c \rightarrow-\infty$ or when $d$ is a constant.

(ii) $\delta(H)>0$ whp when $c \rightarrow+\infty$.

In particular there is no perfect matching in $H$ whp when $c \rightarrow-\infty$.

Sketch of proof. We consider two types of events:

$$
B_{y}^{+}=\{\Gamma(y) \cap B=\emptyset\} \quad \text { for } y \in A
$$

and

$$
B_{z}^{-}=\left\{\Gamma^{-1}(z) \cap A=\emptyset\right\} \quad \text { for } z \in B
$$


We also define the random variables

$$
\begin{aligned}
Q^{+} & =\sum_{y \in A} 1_{B_{y}^{+}}, \\
Q^{-} & =\sum_{z \in B} 1_{B_{z}^{-}} \text {and } \\
Q & =Q^{+}+Q^{-} .
\end{aligned}
$$

The condition $\delta(H)>0$ holds if and only if $Q=0$.

The probability that $B_{y}^{+}$occurs equals

$$
\operatorname{Pr}\left(B_{y}^{+}\right)=\frac{\left(\begin{array}{c}
k n-k d \\
k d
\end{array}\right)}{\left(\begin{array}{l}
k n \\
k d
\end{array}\right)} \quad \text { for all } y \in A,
$$

as the neighbourhood of $y$ is chosen uniformly from all $(k d)$-elements subsets of $Z$. Similarly

$$
\operatorname{Pr}\left(B_{z}^{-}\right)=\frac{\left(\begin{array}{c}
n-k d \\
d
\end{array}\right)}{\left(\begin{array}{l}
n \\
d
\end{array}\right)} \quad \text { for all } z \in B
$$

When $d=o\left(n^{2 / 3}\right)$

$$
\operatorname{Pr}\left(B_{y}^{+}\right), \operatorname{Pr}\left(B_{z}^{-}\right)=\Theta\left(\exp \left(-\frac{k d^{2}}{n}\right)\right)
$$

So

$$
\mathbb{E}(Q)=k d\left(\operatorname{Pr}\left(B_{y}^{+}\right)+\operatorname{Pr}\left(B_{z}^{-}\right)\right)=\Theta\left(k d \exp \left(-\frac{k d^{2}}{n}\right)\right)=\Theta\left(e^{-c}\right) .
$$

This shows that for $d=o\left(n^{2 / 3}\right)$ and $c \rightarrow+\infty, \mathbb{E}(Q)=o(1)$. If $c \rightarrow+\infty$, but $d$ is not $o\left(n^{2 / 3}\right)$ it is easy to check that $\mathbb{E}(Q)=o(1)$. The second conclusion follows.

If $c \rightarrow-\infty$, it is adequate to prove that $\operatorname{Pr}\left(Q^{-}=0\right)=o(1)=\operatorname{Pr}\left(Q^{+}=0\right)$. For this we apply Lemma 19 (Chebyshev's inequality). The upper bound $\operatorname{Var}\left(Q^{-}\right) \leqslant \mathbb{E}\left(Q^{-}\right)$and $\operatorname{Var}\left(Q^{+}\right) \leqslant \mathbb{E}\left(Q^{+}\right)$derived in the proof of Lemma 18 holds as Proposition 25 gives that $\operatorname{Pr}\left(B_{z}^{-} \wedge B_{z^{\prime}}^{-}\right) \leqslant \operatorname{Pr}\left(B_{z}^{-}\right)^{2}$ for $z, z^{\prime} \in Z$ and $\operatorname{Pr}\left(B_{y}^{+} \wedge B_{y^{\prime}}^{+}\right) \leqslant \operatorname{Pr}\left(B_{y}^{+}\right)^{2}$ for $y, y^{\prime} \in Y$.

Having proved the first claim of Theorem 2 we proceed to the second. For $c \geqslant 5 \log (k d)$ we apply Proposition 25 in the way described in the proof of Proposition 22 to get that there is a perfect matching in $H$ whp.

We are only left with showing that when $c \rightarrow+\infty$ and $c \leqslant 5 \log (k d)$ the probability

$$
\operatorname{Pr}(\text { There is no perfect matching in } H \wedge \delta(H)>0)=o(1) \text {. }
$$

This can be done in a very similar way to the proof of Proposition 23. Some amendments have to be made, for example one has to consider problematic pairs $(S, T)$ where $S \subseteq A$ and $T \subseteq B$, whereas in the proof of Theorem 4, we have $S \subseteq A \backslash\{y\}$ where $y$ is some vertex in $A$ and $T \subseteq \Gamma(y)$. 
We conclude the section with a quick explanation as to why our method as presented is not strong enough to yield an (asymptotically) exact expression for the probability that there is a perfect matching in $H$; something that Erdős and Rényi achieved for $B(n, p)$.

As we have seen it is enough to get an asymptotically exact value for the probability $\operatorname{Pr}(\delta(H)=0)$. This is equivalent to none of the events $B_{y}^{+}$or $B_{z}^{-}$occurring. Erdös and Rényi used the inclusion-exclusion principle and exact expressions for the probability of events like

$$
\bigwedge_{y \in S} B_{y}^{+} \wedge \bigwedge_{z \in T} B_{z}^{-}
$$

where $S \subseteq A$ and $T \subseteq B$.

It is hard to obtain exact expressions for the probability of this kind of events because of the lack of independence in choosing the edges in $H$. The switching double counting method can be applied to give upper bounds, which appear to be reasonably sharp. Obtaining lower bounds, like the one in (8), seems to be harder.

\section{Commutative graphs}

In this final section we apply the results obtained in Section 4 to prove Theorem 10.

Proof of Theorem $10(i)$. We show that the upper bound on $d$ implies that Plünnecke's upward condition is violated $w h p$ for all edges in $E\left(X_{h-2}, X_{h-1}\right)$. That is, for $x y \in$ $E\left(X_{h-2}, X_{h-1}\right)$, we show that $w h p$ there is no perfect matching in $G$ from $\Gamma(x)$ to $\Gamma(y)$.

We apply the first part of Theorem 4 with $A=\Gamma(x) \subseteq X_{h-1}=Y$ and $\Gamma(y) \subseteq X_{h}=Z$. Hence $n=\left|X_{h-1}\right|=k^{h-1} m, d^{+}=k d$ and $d^{-}=d$. The parameters satisfy the condition $k d \leqslant n$ as $d \leqslant m$. By Theorem 4 there is no perfect matching in $G$ from $\Gamma(x)$ to $\Gamma(y)$ whp provided that

$$
\frac{k d^{2}}{n}-\log k d=\frac{d^{2}}{k^{h-2} m}-\log k d \rightarrow-\infty .
$$

Since it is easy to see that Theorem $10(i)$ holds for $d=o(\sqrt{m})$ (see Section 5 ), we assume that $d \geqslant m^{1 / 2-\varepsilon}$ for some small $\varepsilon>0$, so that $\log k d \geqslant\left(\frac{1}{2}-\varepsilon\right) \log k m$. The non existence whp of a perfect matching between $\Gamma(x)$ and $\Gamma(y)$ is implied by the condition

$$
\frac{d^{2}}{k^{h-2} m}-\left(\frac{1}{2}-\varepsilon\right) \log k m \rightarrow-\infty .
$$

This is in turn implied by the condition

$$
d \leqslant \sqrt{\frac{1}{3} k^{h-2} m \log k m}
$$

and the proof is concluded.

For Theorem 10 (ii) we rely on Proposition 22. 
Proof of Theorem 10 (ii). For $1 \leqslant j \leqslant h-1$ set $G_{j}^{\prime}=G\left[X_{j-1}, X_{j}, X_{j+1}\right]$ to be the induced subgraph of $G$ on the vertex set $X_{j-1} \cup X_{j} \cup X_{j+1}$. We will calculate the probability that Plünnecke's conditions are not satisfied in $G_{j}^{\prime}$ and then apply a union bound.

Let $x y \in E\left(X_{j-1}, X_{j}\right)$ and $H=G[\Gamma(x), \Gamma(y)]$ be the induced subgraph on $(\Gamma(x), \Gamma(y))$. Then, the probability that Plünnecke's upward condition $(P U)$ is violated for $x y$ is

$\operatorname{Pr}(P U$ is violated for $x y)=\operatorname{Pr}($ There is no perfect matching in $H)$

$$
=O\left(k^{2} d^{2} \exp \left(-\frac{d^{2}}{2 k^{j-1} m}\right)\right),
$$

as we see by applying Proposition 22 and noting that the condition on $c$ is satisfied. There are $k^{j} d m$ such edges and so

$$
\operatorname{Pr}\left(P U \text { is violated in } G_{j}^{\prime}\right)=O\left(k^{j+2} d^{3} m \exp \left(-\frac{d^{2}}{2 k^{j-1} m}\right)\right) .
$$

Let $y z \in E\left(X_{j}, X_{j+1}\right)$ and $I_{j}$ the graph obtained by reversing the direction of the edges of $G_{j}^{\prime}$ (called the inverse of $G_{j}^{\prime}$ ). It is easy to see that Plünnecke's downward condition $(P D)$ for $y z$ in $G_{j}^{\prime}$ is equivalent to Plünnecke's upward condition for $z y$ in $I_{j}$. Similarly

$$
\operatorname{Pr}(P D \text { is violated for } y z)=O\left(d^{2} \exp \left(-\frac{d^{2}}{2 k^{j-1} m}\right)\right) .
$$

There are $k^{j+1} d m$ such edges and so

$$
\operatorname{Pr}\left(P D \text { is violated in } G_{j}^{\prime}\right)=O\left(k^{j+1} d^{3} m \exp \left(-\frac{d^{2}}{2 k^{j-1} m}\right)\right) .
$$

Adding the two probabilities gives

$$
\operatorname{Pr}\left(P U \text { or } P D \text { are violated in } G_{j}^{\prime}\right)=O\left(k^{j+2} d^{3} m \exp \left(-\frac{d^{2}}{2 k^{j-1} m}\right)\right) .
$$

The right hand side is an increasing function of $j$ as $k \geqslant 1$ and so

$$
\begin{aligned}
\operatorname{Pr}(P U \text { or } P D \text { are violated in } G) & \leqslant \sum_{j=1}^{h-1} \operatorname{Pr}\left(P U \text { or } P D \text { are violated in } G_{j}^{\prime}\right) \\
& =O\left(h k^{h+1} d^{3} m \exp \left(-\frac{d^{2}}{2 k^{h-2} m}\right)\right) \\
& =O\left(h k^{h+1} m^{4} \exp \left(-\frac{d^{2}}{2 k^{h-2} m}\right)\right) \\
& =O\left(m^{-1 / 2}\right),
\end{aligned}
$$

when $d \geqslant 3 \sqrt{k^{h-2} m \log \left(h k^{h+1} m\right)}$. 
The bounds on $d$ appearing in Theorem 10 have the same asymptotic order. They can be improved slightly, but as we were not able to obtain matching lower and upper bounds we opted to present a proof as simple as possible. Note also that when $0<k \leqslant 1$ one can obtain estimates on the probability that $G$ is a commutative graph by applying Theorem 10 to the inverse of $G$.

We conclude with some remarks linking the present results with those of [23]. For fixed $m$ and $k$ the lower bound on $d$ provided in Theorem 10 surpasses $m$ for sufficiently large $h$. This is of course not possible and implies that for a given $m$ and $d$ there is a limit to how large $h$ can be taken to be. This reflects the fact that infinite biregular commutative graphs do not exist when $k>1$. It should also be noted that explicit constructions are more economical in $m$ than probabilistic: a path is an infinite commutative graph with augmentation 1 and for integer $k>1$ there exists a commutative biregular graph with 3 layers and augmentation $k$ whose bottom layer is a doubleton.

\section{Acknowledgements}

Both authors would like to thank Oriol Serra for his help and support which were instrumental for the completion of the project. The second author would like to thank Tim Gowers for suggesting using random graphs to construct extremal examples for Plünnecke's inequality and for sharing his insight. He would also like to thank Ben Green and Peter Keevash for helpful suggestions. Finally, the authors want to thank the referee for the careful reading of the manuscript and for the numerous comments provided.

\section{References}

[1] B. Bollobás. A probabilistic proof of an asymptotic formula for the number of labelled regular graphs. European J. Combin., 1(4):311-316, 1980.

[2] B. Bollobás and B.D. McKay. The number of matchings in random regular graphs and bipartite graphs. J. Combin. Theory Ser. B, 41(1):80-91, 1986.

[3] E.R. Canfield and B.D. McKay. Asymptotic enumeration of dense 0-1 matrices with equal row sums and equal column sums. Electron. J. Combin., 12:Research Paper 29, 31 pp. (electronic), 2005.

[4] C. Cooper, A. Frieze, and B. Reed. Random regular graphs of non-constant degree: connectivity and Hamiltonicity. Combin. Probab. Comput., 11(3):249-261, 2002.

[5] C. Cooper, A. Frieze, B. Reed, and O. Riordan. Random regular graphs of nonconstant degree: independence and chromatic number. Combin. Probab. Comput., 11(4):323-341, 2002.

[6] R. Diestel. Graph theory, volume 173 of Graduate Texts in Mathematics. SpringerVerlag, Berlin, third edition, 2005.

[7] P. Erdős and A. Rényi. On random matrices. Magyar Tud. Akad. Mat. Kutató Int. Közl, 8:455-461 (1964), 1964. 
[8] C.J. Everett and P.R. Stein. The asymptotic number of integer stochastic matrices. Discrete Math., 1(1):55-72, 1971/72.

[9] J. Friedman. On the second eigenvalue and random walks in random $d$-regular graphs. Combinatorica, 11(4):331-362, 1991.

[10] J. H. Kim and V. H. Vu. Sandwiching random graphs: universality between random graph models. Adv. Math., 188(2):444-469, 2004.

[11] J.H. Kim, B. Sudakov, and V.H. Vu. On the asymmetry of random regular graphs and random graphs. Random Structures Algorithms, 21:216-224, 2002.

[12] J.H. Kim, B. Sudakov, and V.H. Vu. Small subgraphs of random regular graphs. Discrete Math., 307:1961-1967, 2007.

[13] M. Krivelevich, B. Sudakov, V.H. Vu, and N.C. Wormald. Random regular graphs of high degree. Random Structures Algorithms, 18(4):346-363, 2001.

[14] M. Marcus and H. Minc. A survey of matrix theory and matrix inequalities. Dover Publications Inc., New York, 1992. Reprint of the 1969 edition.

[15] B.D. McKay. Subgraphs of random graphs with specified degrees. In Proceedings of the Twelfth Southeastern Conference on Combinatorics, Graph Theory and Computing, Vol. II (Baton Rouge, La., 1981), volume 33, pages 213-223, 1981.

[16] B.D. McKay. Spanning trees in regular graphs. European J. Combin., 4(2):149-160, 1983.

[17] B.D. McKay. Asymptotics for 0-1 matrices with prescribed line sums. In Enumeration and design (Waterloo, 1982), pages 225-238. Academic Press, Toronto, ON, 1984.

[18] B.D. McKay. Asymptotics for symmetric 0-1 matrices with prescribed row sums. Ars Combin., 19(A):15-25, 1985.

[19] B.D. McKay and X. Wang. Asymptotic enumeration of 0-1 matrices with equal row sums and equal column sums. Linear Algebra Appl., 373:273-287, 2003. Special issue on the Combinatorial Matrix Theory Conference (Pohang, 2002).

[20] B.D. McKay and N.C. Wormald. Asymptotic enumeration by degree sequence of graphs with degrees $o\left(n^{1 / 2}\right)$. Combinatorica, 11(4):369-382, 1991.

[21] M.P. Mineev and A.I. Pavlov. The number of $(0,1)$-matrices with given sums over the rows and columns. Dokl. Akad. Nauk SSSR, 230(2):271-274, 1976.

[22] M.B. Nathanson. Additive number theory: Inverse problems and the geometry of sumsets, volume 165 of Graduate Texts in Mathematics. Springer-Verlag, New York, 1996.

[23] G. Petridis. Plünnecke's inequality. Combin. Probab. Comput., 20(6):921-938, 2011.

[24] H. Plünnecke. Eine zahlentheoretische anwendung der graphtheorie. J. Reine Angew. Math., 243:171-183, 1970.

[25] I.Z. Ruzsa. An application of graph theory to additive number theory. Scientia, Ser. A, 3:97-109, 1989. 
[26] I.Z. Ruzsa. Sumsets and structure. In Combinatorial Number Theory and Additive Group Theory. Springer, New York, 2009.

[27] T. Tao and V.H. Vu. Additive Combinatorics. Cambridge University Press, Cambridge, 2006.

[28] N.C. Wormald. Models of random regular graphs. In Surveys in Combinatorics. Cambridge University Press, Cambridge, 1999. 